The original publication is available at http://www.tandf.co.uk/journals/

\title{
The stability of dipolar gyres on a beta-plane
}

\author{
Steven Herbette ${ }^{a, b, ~}{ }^{*}$, Antoine Hochet $^{b}$, Thierry Huck ${ }^{b}$, Alain Colin de Verdière ${ }^{b}$, Jérémy Collin ${ }^{b}$ \\ and Frank Shillington ${ }^{a}$
}

\begin{abstract}
${ }^{a}$ LMI ICEMASA, Department of Oceanography, University of Cape Town, Rondebosch, South Africa.
b Laboratoire de Physique des Océans (UMR6523 CNRS IFREMER IRD UBO), Université de Bretagne Occidentale, Brest, France.
\end{abstract}

*: Corresponding author : Steven Herbette, email address : steven.herbette@univ-brest.fr

\begin{abstract}
:
When a source-sink dipole forces a fluid on a $\beta$-plane limited by a western boundary, the linear steady solution can be obtained analytically and consists of zonally elongated gyres that extend west of the forcing and close as western boundary currents. The nondimensional parameter $R o_{v}=U /\left(4 \beta a^{2}\right)$ (with $U$ the zonal velocity of the flow and $2 a$ the distance between the source and sink) is used to characterize the nonlinearity of the flow. When $R o$ reaches 0.1 , the numerical shallow-water solution shows that the configuration with the source to the north of the sink becomes unstable, while the reverse configuration remains steady. Indeed, that reverse configuration remains steady for much larger values of the nonlinearity parameter $R o_{\nu}$, and begins to share some of the characteristics of a pure inertial circulation. The asymmetry of the stability properties of the two configurations, also found in the laboratory experiments of Colin de Verdière [Quasigeostrophic flows and turbulence in a rotating homogeneous fluid, 1977], is rationalized herein through the stability properties of the zonal central jet that flows between the source and sink. We consider, in turn, (i) the Kuo's [J. Meteor. 1949, 6, 105122] zero potential vorticity gradient necessary criteria (valid for an infinite zonal jet), (ii) enstrophy budgets and (iii) linear stability analysis of the mean flow. All three methods point out to the enhanced instability of the westward jet. We show that the transition regime has the characteristics of a super critical Hopf bifurcation.
\end{abstract}

Keywords: Beta-plane double-gyre ; Source-sink rotating tank experiments ; Jet instability ; Hopf bifurcation 


\section{Introduction}

The forcing of flows by sources and sinks has a long-standing place in geophysical fluid dynamics, since stretching of the background planetary vorticity readily induces relative vorticity. Hide (1968) discusses such flow behaviour in the presence of viscous dissipation on an $f$-plane. On a rotating sphere, the rotation vector is not parallel to the surface of the fluid, and new effects arise: theTaylorProudman-Poincare theorem states that a slowlymoving fluid column preserves its thickness in the direction parallel to the rotation axis. Therefore, when stretched/compressed by a source/sink forcing, such a fluid column must move towards the equator/pole to conserve its height, and fit between the top and bottom surfaces of the fluid (Schopp and Colin deVerdière 1997), the so-called Sverdrup dynamics in Oceanography. Furthermore, the Ekman pumping at the base of the Ekman layer transforms the wind shear stress at the sea surface into a source-sink forcing for the Ocean interior. In a laboratory experiment, Beardsley (1969) uses this property to simulate directly the large-scale wind stress distribution by rotating a rigid lid at a speed slightly different from the container. The use of localized sources and sinks to force flows on a $\beta$-plane originates from the Stommel et al. (1958) laboratory experiment where fluid is injected at the apex of a pie shape basin, mimicking the source of deep water. The free surface rises in the interior providing the upwelling demanded by the Stommel and Arons (1961) abyssal circulation theory. In response to the rise of the fluid surface, fluid columns stretch in the interior of the basin, which induces poleward motion towards the source. Because of this interior northward circulation, the return flow in thewestern boundary current may have a transport that exceeds that of the source. Here, the choice is made to study the fluid 

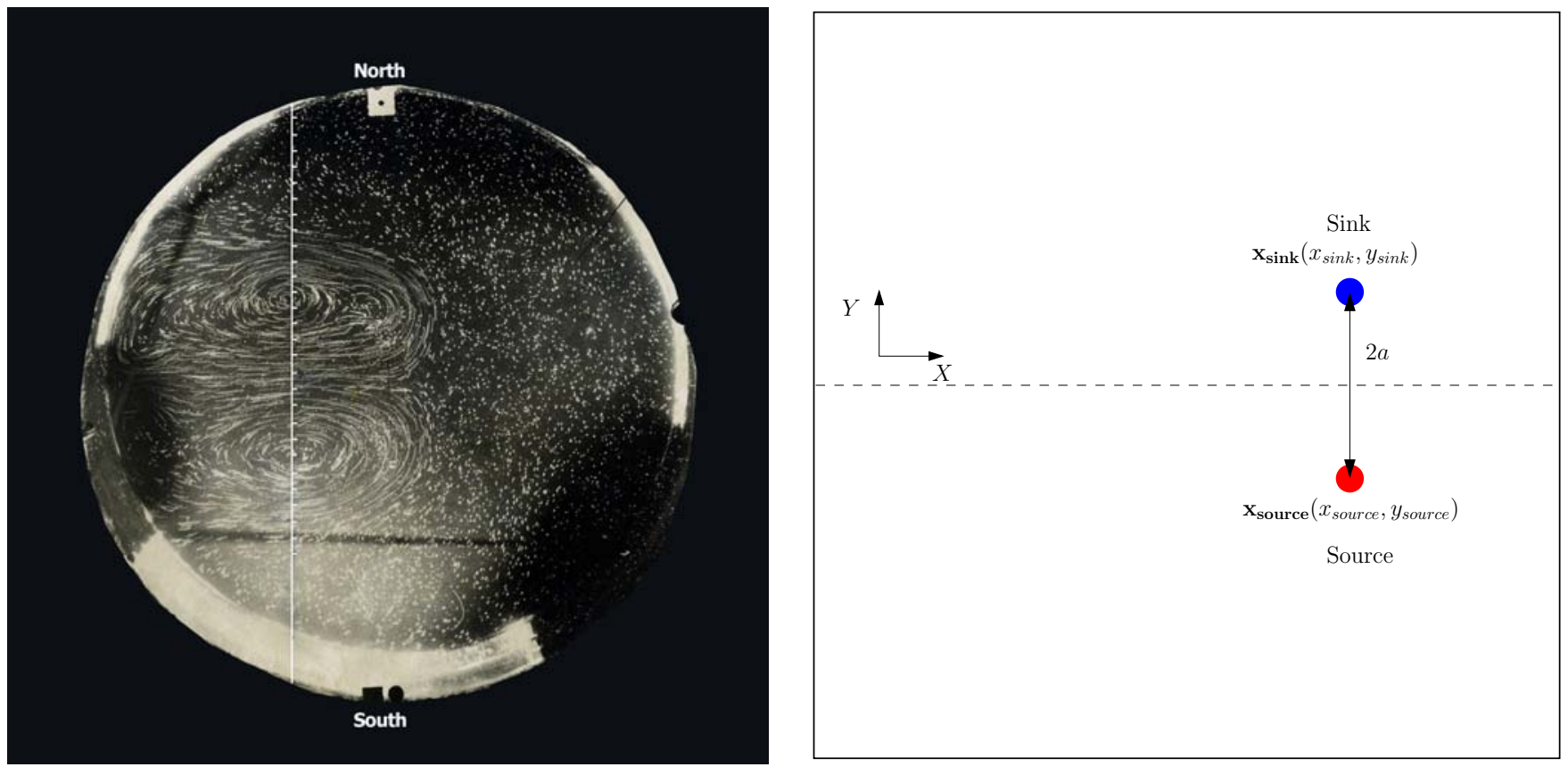

Figure 1. Photograph (Colin de Verdière 1977), at steady state, of a laboratory experiment of a dipolar $\beta$-plane gyre in a rotating cylinder tank (left). Schematic of the experimental set-up used in the following study (right). The bottom rises in the $y$ direction to create the ambient PV gradient in place of the planetary $\beta$-effect. The shallow/deep end of the tank plays the role of the high/low latitudes. Western boundary currents converge to produce a laminar eastward jet that separates the source and sink. Notice the quiescent fluid east of the forcing, and the oblong gyres extending towards the west. In the central region of the jet, some streamlines that are not purely horizontal are also visible. They correspond to beads trapped in the bottom or top Ekman layers and show net fluid exchange from source to sink. [Experimental parameters: the source (south) and sink (north) are separated by $2 a=8 \mathrm{~cm}$, the tank diameter, depth, angular velocity and bottom inclination are: $\left.\mathcal{L}=62 \mathrm{~cm}, H=25 \mathrm{~cm}, \Omega=5.2 \mathrm{rad} \mathrm{s}^{-1}, \alpha=11^{\circ}\right]$.

response to a localized dipole forcing in the form of a Dirac delta function, for a homogeneous flow on the $\beta$-plane. The interior Sverdrup dynamics of such simple flows are described in Pedlosky (1996). Colin de Verdière (1977) presents a laboratory experiment in which he considers the stability of such $\beta$-plane flows in the presence of a western boundary. The flow behaviour depends strongly on the orientation of the dipole in the North-South direction, a puzzling observation that is at the origin of the present paper. At steady state, the dipole generates zonally-elongated $\beta$-gyres extending west of the forcing and closing at the western boundary (figure 1). The Sverdrup balance occurs only locally above the source and sink, and the fluid to the east remains motionless. When the source is to the north (configuration I: the central jet between the source and sink is westward), the flow becomes rapidly unstable with increasing forcing. On the other hand, when the source is to the South (configuration S: the central jet between the source and sink is eastward), the flow remains stable up to very large amplitudes of the forcing [the nonlinearity parameter $U /\left(\beta L^{2}\right)$ is $\mathrm{O}(1)$ ], and the two gyres broaden when the forcing increases. An inviscid argument given in Colin de Verdière (1977) highlights the difficulty of a westward current impinging on a western wall to exit as an interior eastward zonal jet with quiescent fluid outside. Suppose that potential vorticity is conserved and consider the evolution of relative vorticity along the path of a fluid parcel circulating in the northern gyre in the unstable case. Take a parcel that initially lies just north of the line of symmetry. It first moves westward in the central jet, then northward in the western boundary current and finally enters the returning eastward jet. The parcel has gained negative relative vorticity when moving north along the western wall. A vorticity conflict arises when the parcel joins the interior in the external bound of the eastward jet, where positive relative vorticity is required. If the dissipation is too small to resolve this conflict, a transition to unsteady flow is bound to occur. This vorticity conflict does not arise in the stable configuration: a parcel in the external westward jet has negative vorticity that is removed as the parcel moves south along the western wall. It can then join smoothly the central part of the eastward jet with little relative vorticity as it must. Because the asymmetry of the two cases is so clear-cut in the laboratory experiments, the present study aims to find out the causes of their different stability characteristics. Is the primary instability located in the central jets, the external jets or the western boundary? 
Of course this laboratory configuration is far from realistic oceanic configurations, the closest being perhaps Stommel (1982) proposal to interpret the $\beta$-plume distribution of the Helium 3 tracer, west of the Pacific Rise (where it is injected by the hydrovents). At the same time, real fluid experiments with their infinite resolution are ideal tools to test the hydrodynamics of finite resolution numerical codes, their weak points being the difficulty of quantitative measurements. Laboratory and numerical experiments are complementary and the combination allows building up confidence in the analysis of flow experiments. Here, we shall explore the dynamics of the entrance and exit of zonal jets in western boundary current regions, the mixing of potential vorticity in outflows regions, and the inertial stability of zonal jets in the interior, all situations which are obviously important for ocean circulation modelling. In addition, the use of localized forcing in the form of a Dirac delta function suppresses the role of southern and northern boundaries. Away from the forcing, the response of the flow is made of zonally-elongated gyres with zonal jets. Meridional flow are found only at forcing longitudes and at the western boundary. Therefore, we argue that this chosen configuration is the simplest one in order to study the dynamics of zonal flows in the presence of a western boundary using a combination of laboratory and numerical experiments. Since we are discussing de facto the Green function of the oceanic circulation on a $\beta$-plane (albeit in the homogeneous case), we can expect this over-simplified case to be relevant to more general forcing distributions.

The paper is organized as follows. First, we present the governing equations, the experimental setup, and the numerical characteristics of our simulations (section 2). We refer to the nondimensional potential vorticity (PV) equation and introduce an important nondimensional parameter, that we use to interpret our simulations. In section 3, a linear analytical solution is derived and compared to the laboratory experiments. It gives us some insights on the circulation observed at small Rossby numbers, and is used to validate our numerical model. Nonlinear numerical simulations are then performed (section 4). The intriguing asymmetry observed in the laboratory experiments also occurs in the numerical simulations. We use the numerical solutions to get a better knowledge of the circulation, understand the mass transfer from the source to the sink, and infer the role played by the generated eddies (section 5). We then look for an intrinsic dynamical explanation of the observed asymmetry (section 6). We investigate on the spatial origin and type of the instability found in our experiments. We carry out a linear stability analysis of the mean state obtained from the integration of the nonlinear equations to find out the spatial structure of the most unstable mode. Finally, we summarize and discuss our results (section 7 ).

\section{Governing equations, numerical setup, and nondimensional analysis}

\subsection{Governing equations}

Let us consider the dynamics of a rotating, hydrostatic, homogeneous fluid in a rotating square tank of width $\mathcal{L}$ (figure 1). The bottom of the tank rises in the $y$-direction, and the flow is forced by a local dipole, a mass source and sink. The shallow-water equations are:

$$
\begin{aligned}
\partial_{t} h+\boldsymbol{\nabla} \cdot(h \boldsymbol{v}) & =\mathrm{IV}\left(\delta\left(\boldsymbol{x}-\boldsymbol{x}_{\text {source }}\right)-\delta\left(\boldsymbol{x}-\boldsymbol{x}_{\text {sink }}\right)\right), \\
\partial_{t} \boldsymbol{v}+(\boldsymbol{v} \cdot \boldsymbol{\nabla}) \boldsymbol{v}+f_{0} \boldsymbol{k} \times \boldsymbol{v} & =-g \boldsymbol{\nabla} \eta \boldsymbol{D}, \quad f_{0}=2 \Omega .
\end{aligned}
$$

Here $\boldsymbol{v}=(u, v)$ is the horizontal velocity vector, $u$ and $v$ the zonal ( $x$-direction) and meridional ( $y$-direction) velocity component, $h$ the water column thickness, $f_{0}=2 \Omega$ the Coriolis parameter, $\Omega$ the rotating table angular velocity, $g$ the constant for gravity, $\boldsymbol{D}$ the horizontal momentum dissipation, $\boldsymbol{k}$ the vertical unit vector, $\boldsymbol{x}(x, y)$ the horizontal position vector, $\boldsymbol{\nabla}$ the horizontal gradient operator, $\delta$ the Dirac function, and $\mathrm{I}_{\mathrm{V}}$ the rate at which fluid is locally injected in/pumped out of tank. To ensure conservation of mass within our domain, the pumping and injection rates are of equal intensity. We set them symmetrically relative to $y=0$ axis to respect the geometrical constraints of our domain and ensure that the anticyclonic and cyclonic gyre have similar shape. The positions of the source and sink are the following: $\boldsymbol{x}_{\text {source }}=\left(x_{s},+a\right)$, $\boldsymbol{x}_{\text {sink }}=\left(x_{s},-a\right)$. The x-position of the dipole is kept constant in our setup: $x_{s}=2 / 3 \mathcal{L}$, while sensitivity experiments are carried out varying $2 a$, the distance that separates the source from the sink. The water 
Table 1. Parameters of the experiments. $\mathcal{L}$ : width the square tank; $H$ : average depth; $\alpha$ : angle of the bottom slope with the horizontal; $\tau$ : rotating period; $\mathrm{I}_{\mathrm{V}}$ : rate at which fluid is injected in/pumped out at the source/sink; $2 a$ : distance between the source and the sink. To limit spurious numerical effects, the fluid is injected in/pumped out over an area $\mathcal{A}_{\text {source/sink }}$, rather than at a single grid point. $\lambda$ is a fundamental nondimensional parameter describing the linear analytical solution.

\begin{tabular}{ccccccccccc}
\hline $\begin{array}{c}H \\
(\mathrm{~cm})\end{array}$ & $\begin{array}{c}\mathcal{L} \\
(\mathrm{m})\end{array}$ & $\begin{array}{c}\alpha \\
\left({ }^{\circ}\right)\end{array}$ & $\begin{array}{c}\tau_{\text {tank }} \\
(\mathrm{s})\end{array}$ & $\begin{array}{c}f_{0} \\
\left(\mathrm{~s}^{-1}\right)\end{array}$ & $\begin{array}{c}\mathrm{I}_{\mathrm{V}} \\
\left(\mathrm{ml} \mathrm{min}^{-1}\right)\end{array}$ & $\begin{array}{c}\nu \\
\left(\mathrm{m}^{2} \mathrm{~s}^{-1}\right)\end{array}$ & $\begin{array}{c}\mathrm{r} \\
\left(\mathrm{s}^{-1}\right)\end{array}$ & $\begin{array}{c}\mathcal{A}_{\text {source } / \text { sink }} \\
\left(\mathrm{cm}^{2}\right)\end{array}$ & $\begin{array}{c}2 a \\
(\mathrm{~cm})\end{array}$ & $\lambda=\beta_{T} \mathcal{L} /(2 r)$ \\
\hline 36.93 & 0.5 & 7 & 8 & 1.57 & {$[1,400]$} & $10^{-6}$ & $6 \cdot 10^{-3}$ & 1 & {$[5,40]$} & 22 \\
\hline
\end{tabular}

column thickness $h$ is expressed as:

$$
h(x, y)=H+\eta(x, y)-s y
$$

with $s=\tan \alpha$ the bottom slope, $\eta$ the surface displacement, and $H$ the average depth at rest. With this choice of topography, the $y$ axis is orientated towards the high latitudes, i.e. the north for a northern hemisphere setup (see Greenspan 1969 for a presentation of the topographic analogue to the planetary $\beta$ ).

\section{$2.2 \quad$ Numerical setup and parameters}

Equations (1a,b) are integrated in time using the Miami Isopycnal Coordinate Ocean Model (MICOM) (Bleck and Boudra 1986, Bleck et al. 1992) in a shallow-water version that has been adapted for this study. As in Herbette et al. (2003), the nonlinear terms of the momentum equations are solved with a fourth-order centred finite difference scheme. Dissipation is ensured through linear friction (parametrizing the bottom Ekman layer) and Newtonian harmonic dissipation:

$$
\boldsymbol{D}=-r \boldsymbol{v}+\nu \nabla^{2} \boldsymbol{v}
$$

with $r$ and $\nu$ the coefficients for the linear bottom friction and water kinematic viscosity. To stay as close as possible to the laboratory experiments, we set the numerical viscosity coefficient to the molecular value of sea-water: $\nu=10^{-6} \mathrm{~m}^{2} \mathrm{~s}^{-1}$. The linear bottom friction coefficient is set to $r=6 \times 10^{-3} \mathrm{~s}^{-1}$, a value of the order of the inverse of the spindown time due to the control of the geostrophic interior by the bottom Ekman layers (Greenspan 1969). The introduction of viscous effects introduces a subtle choice for the numerical boundary condition at the wall. In the following, because we wish to model laminar viscous flows in a tank, we use the no-slip boundary condition. Some sensitivity experiments have also been carried out with the slip lateral boundary condition. The general characteristics of the flow have not been affected.

In order to show the exchange of water masses between the two gyres, a passive tracer is also injected at the source. The evolution of the tracer concentration obeys the following equation:

$$
\partial_{t}(h C)+\boldsymbol{\nabla} \cdot(h C \boldsymbol{v})=\mathrm{IV}_{\mathrm{V}}\left(C_{\text {source }} \delta\left(\boldsymbol{x}-\boldsymbol{x}_{\text {source }}\right)-C \delta\left(\boldsymbol{x}-\boldsymbol{x}_{\text {sink }}\right)\right),
$$

with $C(\boldsymbol{x}, t)$ the local concentration at time $t$, and $C_{\text {source }}=1$ at the source. Equation (4) is solved using the same flux corrected transport scheme that is used to solve the continuity equation (Zalesak 1979). Parameters of the experiments are summarized in table 1. Horizontal resolution is $2 \mathrm{~mm}$ and time step $2 \times 10^{-4} \mathrm{~s}$. Each experiment is run for at least 400 rotating periods, i.e. 100 periods more than it takes for the over whole energy and potential vorticity to reach a statistical equilibrium.

\subsection{Potential vorticity equation and nondimensional analysis}

The potential vorticity, hereinafter referred to as PV, often occurs to be a very useful quantity in the analysis of geophysical rotating flows. In the absence of diabatic forcing and dissipation, fluid particles conserve their PV. Using the quasi-geostrophic framework, PV can be inverted to infer the circulation of the flow (Hoskins et al. 1985). Nevertheless, PV is not a good indicator of the circulation within eddies, 
because it is often dominated by the ambient PV related to the variation of the Coriolis parameter with latitude or topography. In the following, we prefer to use potential vorticity anomaly, hereafter referred to as PVA or $Q$ in the equations (Morel and McWilliams 2001, Herbette et al. 2003, 2005):

$$
P V=\frac{\zeta+f_{0}}{h}, \quad P V A \equiv Q=H\left(\frac{\zeta+f_{0}}{h}-\frac{f_{0}}{H}\right)
$$

with $\zeta=\boldsymbol{k} \cdot \boldsymbol{\nabla} \times \boldsymbol{v}$ the relative vorticity. PVA gives better insights on the dynamics of the flow. It has the same conservation property as PV and can be used as a good proxy for the strength and polarity of the circulation (Herbette et al. 2004). Anticyclonic/cyclonic circulations are thus mostly associated with negative/positive PVA. Additionally, in the case of weak Rossby numbers and weak topographic slope, PVA relates directly to the quasi-geostrophic expression of $\mathrm{PV}^{1}$. The equation for the local evolution of PVA, is derived by taking the curl of (1b) and using (1a):

$$
\left(\partial_{t}+\boldsymbol{v} \cdot \nabla\right) Q=H\left[-\frac{r}{h} \zeta+\frac{\nu}{h} \nabla^{2} \zeta-\mathrm{I}_{\mathrm{V}}\left(\frac{\zeta+f_{0}}{h^{2}}\right)\left(\delta\left(\boldsymbol{x}-\boldsymbol{x}_{\text {source }}\right)-\delta\left(\boldsymbol{x}-\boldsymbol{x}_{\text {sink }}\right)\right)\right]
$$

In the limit of no dissipation $(\boldsymbol{D}=\mathbf{0})$ and no forcing $\left(\mathrm{I}_{\mathrm{V}}=0\right)$, fluid particles conserve their PVA. Injecting/pumping out fluid at a source/sink generates positive/negative vertical velocities, which creates negative/positive PVA, respectively associated with anticyclonic/cyclonic circulation. In the following experiments, the vortex stretching terms associated with the surface elevation are small: $f_{0}^{2} L^{2} /(g H) \simeq$ $4 \times 10^{-2}$, with $L=\mathcal{L} / 2$. Therefore, the flow behaves as in the rigid lid experiments of Colin de Verdière (1977). If the bottom slope is also small $(s \ll H / \mathcal{L})$, the expression for the PVA becomes:

$$
Q \simeq \zeta+\beta_{T} y, \quad \text { with } \quad \beta_{T}=\frac{f_{0} s}{H} .
$$

For small Rossby numbers, small topography and rigid lid, the governing equation for $Q$ reduces to the classic barotropic quasi-geostrophic vorticity equation (Greenspan 1969):

$$
\partial_{t} \zeta+u \partial_{x} \zeta+v \partial_{y} \zeta+\beta_{T} v=-r \zeta+\nu \nabla^{2} \zeta-\frac{f_{0} \mathrm{I}_{\mathrm{V}}}{H}\left(\delta\left(\boldsymbol{x}-\boldsymbol{x}_{\text {source }}\right)-\delta\left(\boldsymbol{x}-\boldsymbol{x}_{\text {sink }}\right)\right),
$$

where $\zeta=\nabla^{2} \Psi$, and $\Psi=g \eta / f_{0}$ the stream function. The latter equation is nondimensionalised using $L$ as a length scale, $U$ as a horizontal velocity and introducing classic length scales that emerge when considering the dynamics of a basin gyre, respectively the Stommel $\left(\delta_{S}\right)$, the Munk $\left(\delta_{M}\right)$ and the inertial $\left(\delta_{I}\right)$ boundary layer thickness (Stommel 1948, Munk 1950, Charney 1955):

$$
\delta_{S}=\frac{r}{\beta_{T}}, \quad \delta_{M}=\left(\frac{\nu}{\beta_{T}}\right)^{\frac{1}{3}}, \quad \delta_{I}=\sqrt{\frac{U}{\beta_{T}}} .
$$

When the vortex stretching terms are neglected, eight physical parameters govern the flow: the average fluid depth at rest $H$, the length of the tank $\mathcal{L}$, the bottom slope $\alpha$, the Coriolis parameter $f_{0}$, the distance separating the source and sink $2 a$, the rate of volume injection and pumping $\mathrm{I}_{\mathrm{V}}$, the linear bottom friction coefficient $r$, and the molecular viscosity $\nu$. They can be reduced and combined into seven nondimensional parameters, of which three relate to the geometry of the experimental setup $(H / L, \alpha, 2 a / L)$ and four

${ }^{1}$ For small variations of the layer thickness, the shallow-water expression of the PV writes as

$$
Q=\left(\zeta+f_{0}\right)\left(1-\frac{\eta}{H}+\frac{s y}{H}\right)
$$

and for small Rossby numbers it becomes the familiar quasi-geostrophic expression of PV:

$$
Q_{Q G}=\zeta-f_{0} \frac{\eta}{H}+f_{0} \frac{s y}{H} .
$$


to the dynamics of the flow $\delta_{S} / L, \delta_{M} / L, \delta_{I} / L,\left(f_{0} \mathrm{I}_{\mathrm{V}}\right) /\left(\beta_{T} U H L^{2}\right)$. The latter has been used in previous studies dealing with wind-driven gyres to estimate the horizontal velocity (Marshall 1984, Moro 1988, Cessi et al. 1990, Fox-Kemper and Pedlosky 2004):

$$
U=\frac{f_{0} \mathrm{I}_{\mathrm{V}}}{H \mathcal{L}^{2} \beta_{T}}
$$

Note that (9) is just a Sverdrup scaling of the meridional velocity in which $\mathrm{I}_{\mathrm{V}}$ could be be interpreted as the scale of the integrated Ekman pumping over an ocean basin. Because the zonal velocity found in our experiments is much larger than this, in order to characterize our simulations, $U$ is instead estimated taking the maximum amplitude of the mean zonal jet obtained after numerical integration. Equation (7) is finally re-written in its nondimensional form as follows:

$$
\frac{\delta_{I}^{2}}{L^{2}}\left(\partial_{t} \zeta+u \cdot \partial_{x} \zeta+v \cdot \partial_{y} \zeta\right)+v=-\frac{\delta_{S}}{L} \zeta+\frac{\delta_{M}^{3}}{L^{3}} \nabla^{2} \zeta-\left(\delta\left(\boldsymbol{x}-\boldsymbol{x}_{\text {source }}\right)-\delta\left(\boldsymbol{x}-\boldsymbol{x}_{\text {sink }}\right)\right)
$$

We choose to stay in the regime of parameters tested in the laboratory experiments of Colin de Verdière (1977) and carry out some sensitivity experiments varying the intensity of the forcing and the distance between the source and the sink. The values chosen for $\mathcal{L}, f_{0}, \alpha, r, \nu$ (table 1 ) ensure that the widths of the Stommel $\left(\delta_{S}\right)$ and the Munk $\left(\delta_{M}\right)$ boundary layers are similar and well resolved within our simulations: $\delta_{S}=1.24 \mathrm{~cm}, \delta_{M}=1.28 \mathrm{~cm}$. Several choice can be made for the typical length scale $L$. To measure the degree of inertia of our simulations and the importance of the nonlinear terms in (10), we use the source-sink distance $2 a$ as the length scale to compute the Rossby number of the vorticity equation:

$$
R o_{v}=\frac{\delta_{I}^{2}}{L^{2}}=\frac{U}{\beta L^{2}}=\frac{U}{4 \beta_{T} a^{2}}
$$

$R o_{v}$ is used to differentiate and interpret our experiments. It also provides an estimate of the ratio of the meridional gradient of the central jet's relative vorticity over the ambient potential vorticity, and therefore relates to the Kuo (1949) criteria for barotropic instability of zonal jets.

The nondimensional parameters of the different experiments are summarized in table 2. According to the chosen pumping/injection rate, the width of the inertial boundary layer varies roughly between $0.9 \mathrm{~cm}$ and $12.0 \mathrm{~cm}$. In addition, for each set of parameters, two experiments are considered, one with the source located poleward of the sink (configuration I), and one with the source located southward of the sink (configuration S).

\section{Linear analytical solution}

\subsection{Analytic: the $\beta$ plume solution with a western boundary}

For weak pumping and injection rate, fluid velocities and free surface elevations are small enough to consider a steady linear analytical solution. Equation (10) may be linearised for small ratios of the width of the inertial and Munk boundary layers over the width of the Stommel layer: $\left(R o_{v}, \delta_{M}^{3} / L^{3}\right) \ll \delta_{S} / L$. After defining a nondimensional streamfunction $\Psi$ for the horizontal flow, (10) can be re-written as follows:

$$
\begin{aligned}
\nabla^{2} \Psi+2 \lambda \frac{\partial \Psi}{\partial x} & =-\left(\delta\left(\boldsymbol{x}-\boldsymbol{x}_{\text {source }}\right)-\delta\left(\boldsymbol{x}-\boldsymbol{x}_{\text {sink }}\right)\right) \\
2 \lambda & =\frac{L}{\delta_{S}}, \quad \partial_{x} \Psi=v, \quad \partial_{y} \Psi=-u, \quad \nabla^{2} \Psi=\zeta .
\end{aligned}
$$


Table 2. Numerical experiments run with the no-slip lateral boundary conditions in configuration I and S. We report the maximum amplitude of the time-average zonal velocity within the central jet $\left(x_{\text {source }}-0.18 \mathrm{~m}<x<x_{\text {source }}-0.08 \mathrm{~m}\right)$. EDDY and FRIC refer respectively to the role played by the intergyre s' eddy vorticity fluxes, and bottom friction in dissipating the input of vorticity within each gyre.

\begin{tabular}{|c|c|c|c|c|c|c|c|}
\hline \multirow[b]{2}{*}{$\begin{array}{c}\mathrm{I}_{\mathrm{V}} \\
\left(\mathrm{ml} \mathrm{min}^{-1}\right)\end{array}$} & \multirow[b]{2}{*}{$\begin{array}{c}2 a \\
(\mathrm{~cm})\end{array}$} & \multicolumn{3}{|c|}{ Configuration I } & \multicolumn{3}{|c|}{ Configuration S } \\
\hline & & $\begin{array}{c}\max \left[\left|\bar{u}_{\text {jet }}\right|\right] \\
\left(\mathrm{mm} \mathrm{s}^{-1}\right)\end{array}$ & $R o_{v}$ & $\begin{array}{c}\text { Gyre Vorticity } \\
\text { Balance }\end{array}$ & $\begin{array}{c}\max \left[\left|\bar{u}_{\text {jet }}\right|\right] \\
\left(\mathrm{mm} \mathrm{s}^{-1}\right)\end{array}$ & $R_{v}$ & $\begin{array}{c}\text { Gyre Vorticity } \\
\text { Balance }\end{array}$ \\
\hline 1 & 10 & 0.02 & 0.004 & & 0.02 & 0.004 & \\
\hline 5 & 10 & 0.11 & 0.02 & & 0.11 & 0.02 & \\
\hline 10 & 10 & 0.23 & 0.04 & & 0.23 & 0.04 & \\
\hline 30 & 10 & 0.68 & 0.13 & $\begin{array}{l}E D D Y \simeq 0 \% \\
F R I C \simeq 60 \%\end{array}$ & 0.64 & 0.12 & $\begin{array}{l}E D D Y \simeq 0 \% \\
F R I C \simeq 60 \%\end{array}$ \\
\hline 50 & 10 & 1.06 & 0.20 & $\begin{array}{l}E D D Y \simeq 1 \% \\
F R I C \simeq 55 \%\end{array}$ & 1.07 & 0.20 & $\begin{array}{l}E D D Y \simeq 0 \% \\
F R I C \simeq 58 \%\end{array}$ \\
\hline 100 & 10 & 1.65 & 0.32 & $\begin{array}{l}E D D Y \simeq 19 \% \\
F R I C \simeq 41 \%\end{array}$ & 2.22 & 0.43 & $\begin{array}{l}E D D Y \simeq 0 \% \\
F R I C \simeq 61 \%\end{array}$ \\
\hline 100 & 20 & 1.68 & 0.08 & & 1.39 & 0.06 & \\
\hline 100 & 30 & 1.12 & 0.02 & & 0.43 & 0.01 & \\
\hline 200 & 10 & 2.86 & 0.54 & $\begin{aligned} E D D Y & \simeq 29 \% \\
F R I C & \simeq 34 \%\end{aligned}$ & 4.33 & 0.83 & $\begin{array}{l}E D D Y \simeq 1 \% \\
F R I C \simeq 64 \%\end{array}$ \\
\hline
\end{tabular}
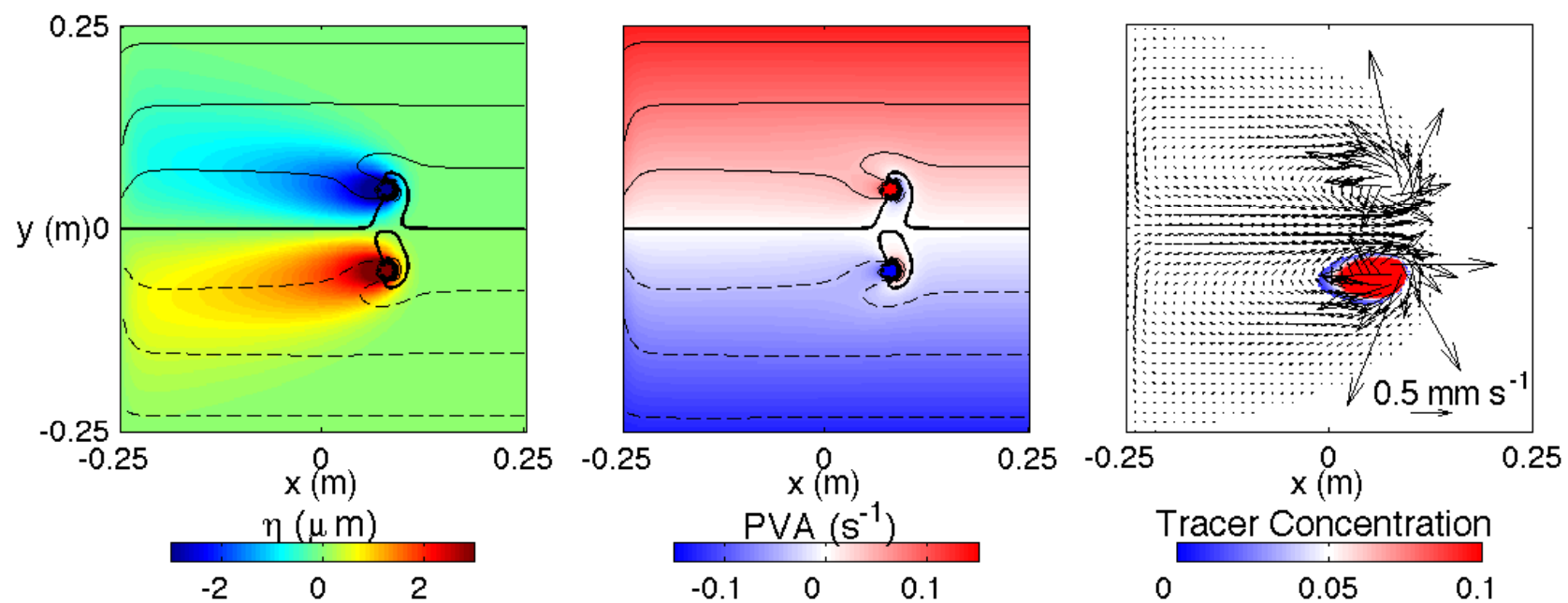

Figure 2. Linear analytical solution of the sea surface elevation $\left(\eta=f_{0} / g \Psi\right.$, left), PVA $\left(\nabla^{2} \Psi+\beta_{T} y\right.$, middle), and velocity vectors $\left(u=-\partial_{y} \Psi, v=\partial_{x} \Psi\right.$, right) for a dipolar gyre forced by a localized source and sink in configuration $\mathrm{S}$. IV $=10 \mathrm{ml} \mathrm{min}^{-1}, 2 a=10 \mathrm{~cm}$, $R o_{v} \simeq 0.04$. The localized source and sink respectively force an anticyclonic and cyclonic gyre, both with intensified western boundary current. In configuration I, (not shown), the linear analytical solution is the exact anti-symmetric relative to the $y=0$ axis [see equation (22)]. This regime is valid for $R o_{v} \precsim 0.1$. The distribution of tracer, from the numerical integration, at $t=400 \tau_{\text {tank }}$, is superimposed on the right panel. Thin solid/dashed contours are for positive/negative values. Contour interval is $0.04 \mu \mathrm{m}$ for $\eta$ and $0.04 \mathrm{~s}^{-1}$ for PVA. Colour version online.

We look for the Green functions $G\left(\boldsymbol{x}, \boldsymbol{x}^{\prime}\right)$ of (12) solution of:

$$
\nabla^{2} G+2 \lambda \frac{\partial G}{\partial x}=\delta\left(\boldsymbol{x}-\boldsymbol{x}^{\prime}\right)
$$


Equation (14) can be transformed to a Helmholtz equation by writing $G\left(\boldsymbol{x}, \boldsymbol{x}^{\prime}\right)=\exp \left(-\lambda\left(x-x^{\prime}\right)\right) \mathcal{G}\left(\boldsymbol{x}, \boldsymbol{x}^{\prime}\right)$ :

$$
\nabla^{2} \mathcal{G}-\lambda^{2} \mathcal{G}=\exp \left(+\lambda\left(x-x^{\prime}\right)\right) \delta\left(\boldsymbol{x}-\boldsymbol{x}^{\prime}\right)
$$

We can switch to polar coordinates $(R, \theta)$ with the origin at the source point $\left(x^{\prime}, y^{\prime}\right): R=$ $\sqrt{\left(x-x^{\prime}\right)^{2}+\left(y-y^{\prime}\right)^{2}}$. Equation (15) becomes:

$$
R^{2} \frac{\partial^{2} \mathcal{G}}{\partial R^{2}}+R \frac{\partial \mathcal{G}}{\partial R}-R^{2} \lambda^{2} \mathcal{G}=\exp \left(+\lambda\left(x-x^{\prime}\right)\right) \delta(R)
$$

This is a modified Bessel equation of the second kind whose solution bounded at infinity is:

$$
\mathcal{G}(R)=\frac{1}{2 \pi} \mathrm{K}_{0}(\lambda R)
$$

This leads to:

$$
G\left(\boldsymbol{x}, \boldsymbol{x}^{\prime}\right)=\frac{1}{2 \pi} \exp \left(-\lambda\left(x-x^{\prime}\right)\right) \mathrm{K}_{0}\left(\lambda \sqrt{\left(x-x^{\prime}\right)^{2}+\left(y-y^{\prime}\right)^{2}}\right) .
$$

This so-called $\beta$ plume solution is the response to a $\delta$ function on an infinite $\beta$-plane (Pedlosky 1996). However, this solution does not satisfy the no-flow condition at the western and eastern boundaries. Assuming an infinite domain in the y-direction and a western boundary with no normal flow, the Green function $G_{2}\left(\boldsymbol{x}, x^{\prime}\right)$ may be found by using the method of images (Morse and Feschbach 1953). Let us consider the case of an interior source of intensity unity located at point $\left(x^{\prime}, y^{\prime}\right)$, and its symmetric image with respect to the western boundary, a sink of intensity $\mathrm{I}_{i m}$ at point $\left(x_{i m}^{\prime}, y_{i m}^{\prime}\right) . G_{2}$ is just the linear superposition of the solutions obtained considering the source and its image separately:

$$
\begin{aligned}
G_{2}\left(\boldsymbol{x}, \boldsymbol{x}^{\prime}\right)= & \frac{1}{2 \pi} \exp \left(-\lambda\left(x-x^{\prime}\right)\right) \mathrm{K}_{0}\left(\lambda \sqrt{\left(x-x^{\prime}\right)^{2}+\left(y-y^{\prime}\right)^{2}}\right) \ldots \\
& -\frac{\mathrm{I}_{i m}}{2 \pi} \exp \left(-\lambda\left(x-x_{i m}^{\prime}\right)\right) \mathrm{K}_{0}\left(\lambda \sqrt{\left(x-x_{i m}^{\prime}\right)^{2}+\left(y-y_{i m}^{\prime}\right)^{2}}\right) .
\end{aligned}
$$

To find $\mathrm{I}_{i m}$ and $x_{i m}^{\prime}$, we require that the normal velocity at the western boundary vanishes : $G_{2}\left(x_{w}, y\right)=0$ with $x_{w}=-\mathcal{L} / 2$. This leads to:

$$
x_{i m}^{\prime}=2 x_{w}-x^{\prime}, \quad y_{i m}^{\prime}=y^{\prime}, \quad \mathrm{I}_{i m}=-\exp \left(-2 \lambda\left(x_{w}-x^{\prime}\right)\right) .
$$

The final Green function solution of (14) with a western boundary is:

$$
G_{2}\left(\boldsymbol{x}, \boldsymbol{x}^{\prime}\right)=\frac{1}{2 \pi} \exp \left(-\lambda\left(x-x^{\prime}\right)\right)\left(\mathrm{K}_{0}\left(\lambda \sqrt{\left(x-x^{\prime}\right)^{2}+\left(y-y^{\prime}\right)^{2}}\right)-\mathrm{K}_{0}\left(\lambda \sqrt{\left(x+x^{\prime}-2 x_{w}\right)^{2}+\left(y-y^{\prime}\right)^{2}}\right)\right),
$$

and the final solution of (10), with a source and a sink, is:

$$
\begin{aligned}
\Psi(x, y)=\frac{1}{2 \pi} \exp \left(-\lambda\left(x-x_{\text {source } / \text { sink }}\right)\right)( \\
\mathrm{K}_{0}\left(\lambda \sqrt{\left(x-x_{\text {source }}\right)^{2}+\left(y-y_{\text {source }}\right)^{2}}\right)-\mathrm{K}_{0}\left(\lambda \sqrt{\left(x+x_{\text {source }}\right)^{2}+\left(y-y_{\text {source }}\right)^{2}}\right) \\
\left.-\mathrm{K}_{0}\left(\lambda \sqrt{\left(x-x_{\text {sink }}\right)^{2}+\left(y-y_{\text {sink }}\right)^{2}}\right)+\mathrm{K}_{0}\left(\lambda \sqrt{\left(x+x_{\text {sink }}\right)^{2}+\left(y-y_{\text {sink }}\right)^{2}}\right)\right) .
\end{aligned}
$$


The dimensional form of $\Psi$ is obtained by multiplying (22) by $\left(f_{0} \mathrm{I}_{\mathrm{V}}\right) /(H r)$ and replacing $\lambda$ by its dimensional form $\lambda^{*}=\beta_{T} /(2 r)$. The sole parameter $\lambda$ governs the solution and is large in geophysical applications; here $\lambda \simeq 22$ taking $L=\mathcal{L}$.

\subsection{Description of the linear solution and comparison with the numerical solution}

Equation (22) provides an analytical expression for the streamfunction $\Psi$ of a double-gyre circulation forced by a localized source and sink on a $\beta$-plane. This solution is only valid with one western boundary. But given the asymptotic decay of the solution east of the Dirac delta forcing, this is also an approximate solution in a closed basin if the eastern boundary is far enough, that is for large $\lambda$. The corresponding quasi-geostrophic potential vorticity anomaly, velocity components and free surface elevation are shown in figure 2 . The analytical solution looks very much like the circulation obtained in the laboratory experiments of Colin de Verdière (1977) for Rossby number up to $R o_{v} \simeq 0.1$ in configuration I, and $R o_{v} \simeq 0.5$ in configuration S. As expected, the sea surface elevation rises around the source, and lowers around the sink, which creates respectively an anticyclonic and cyclonic circulation. The PVA field confirms this general picture of the flow, with a negative/positive patch of PVA around the source/sink. The iso-lines of PVA stay mostly parallel to the iso-depths, with values increasing towards the shallow depths. The circulation is shaped into two basin oblong symmetric gyres. Intensified western boundary currents close the circulation. Around the centre of the basin, a strong zonal jet separates the two gyres. This jet flows westward in configuration I and eastward in configuration S. Note that for the linear solution, interchanging the position of the source and sink just reverses the flow within the gyres, but does not change the shape of the gyres, nor their intensity. We run the numerical model for 400 rotating periods for weak pumping/injection rates in a range of $\mathrm{I}_{\mathrm{V}}=[1,10] \mathrm{ml} \mathrm{min}^{-1}\left(R o_{v} \simeq[0.004,0.04]\right)$ with a source and a sink separated by $10 \mathrm{~cm}$. For the weak inertial flow considered here, a steady state is reached in less than 100 rotating periods. That steady state (not shown) is very similar to the analytical solution (figure 2). For the spatial resolution that is considered $(\delta x=2 \mathrm{~mm})$, there is less than $5 \%$ discrepancies ${ }^{1}$ when the pumping/injection rate is $\mathrm{I}_{\mathrm{V}}=5 \mathrm{ml} \mathrm{min}{ }^{-1}$, and less than $10 \%$ discrepancies when it reaches $\mathrm{I}_{\mathrm{V}}=10 \mathrm{ml} \mathrm{min}^{-1}$. Our ability to represent accurately the dissipation processes taking place in the Stommel layer (6 grids points in the western boundary layer) is crucial. The main discrepancies occur in a very tight region situated around the source and sink, and to a lesser extent near the western boundary where viscous effects start to influence the numerical solution.

\section{Exploring the nonlinear regime and the transition from a stable to an unstable oscillating solution}

For rates higher than $30 \mathrm{ml} \mathrm{min}{ }^{-1}$, the intensity of the circulation increases, so as the width of the inertial boundary layer $\delta_{I}$. When the latter reaches the width of the Munk and Stommel boundary layer, the linear analytical solution loses its validity, in particular near the western boundary. Hence, we explore this regime carrying out numerical experiments with forcing rates varying from $\mathrm{I}_{\mathrm{V}}=30 \mathrm{ml} \mathrm{min}^{-1}\left(R o_{v} \simeq 0.1\right)$ to $\mathrm{I}_{\mathrm{V}}=400 \mathrm{ml} \mathrm{min}^{-1}\left(R o_{v} \simeq 1.5\right)$. The different simulations and their associated parameters are summarized in table 2. The steady state is deduced from plotting time series of volume integrated Kinetic Energy $\left(\mathrm{KE}_{V}\right)$ and Potential Vorticity $\left(Q_{V}\right)$ (figure 3 ). For moderate nonlinear flows (Rov $\precsim 0.2$ ), interchanging the position of the source and sink has no impact, neither on the mean level of kinetic energy of the double gyre system, nor on the total PV embedded within each gyre. As expected, $\mathrm{KE}_{V}$ increases with increasing pumping/injection rate, so as the intensity of the gyre's circulation. Above some threshold value of the forcing, $\mathrm{KE}_{V}$ and $Q_{V}$ start to oscillate around a mean value and the symmetry between

${ }^{1}$ The root mean square discrepancy, $e$, between the analytical and the numerical solution is estimated as follows:

$$
e=100 \sqrt{\frac{1}{\int_{\mathcal{D}} \mathrm{d} A} \int_{\mathcal{D}} \frac{\left(\eta_{\mathrm{th}}(x, y)-\eta_{\mathrm{num}}(x, y)\right)^{2}}{\max \left[\left|\eta_{\mathrm{th}}(x, y)\right|,\left|\eta_{\operatorname{num}}(x, y)\right|\right]^{2}} \mathrm{~d} A},
$$

$\eta_{\mathrm{th}}$ and $\eta_{\text {num }}$ are the theoretical and numerical free surface elevation. Grid points with $\eta<0.1 \mu \mathrm{m}$ have not been considered when computing the spatial integrals over the horizontal domain $\mathcal{D}$. 

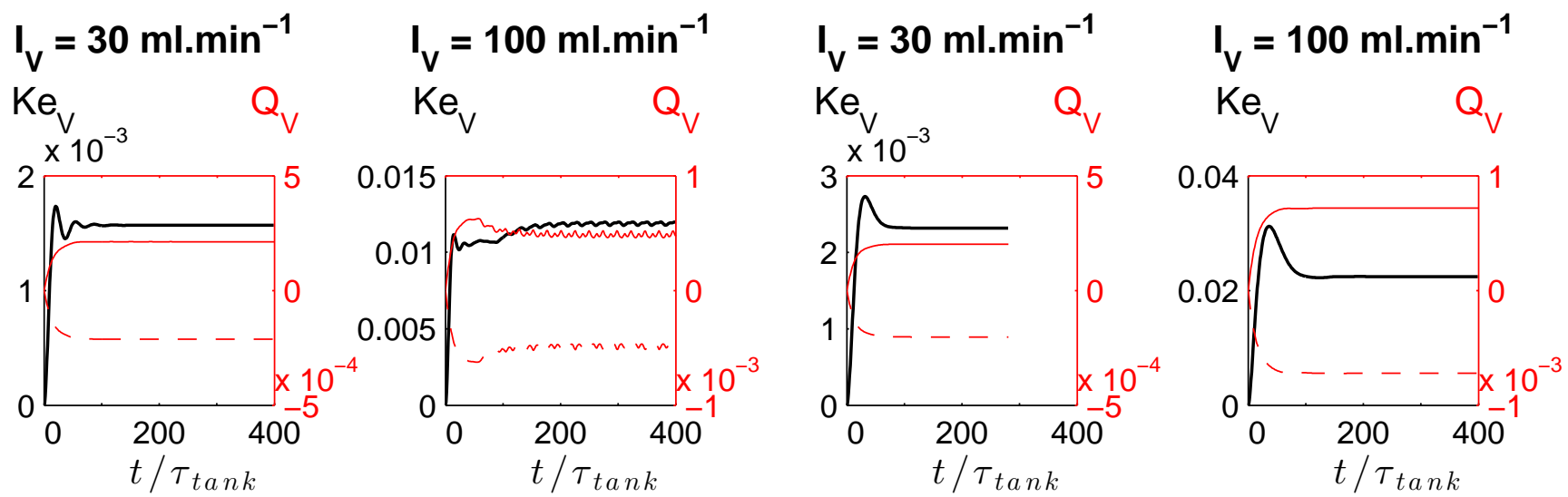

Figure 3. Time series of volume integrated kinetic energy (black, left y-axis) and potential vorticity (red, right y-axis) for configuration I (first two columns) and $\mathrm{S}$ (last two columns). $\mathrm{I}_{\mathrm{V}}=30 \mathrm{ml} \mathrm{min}^{-1}$ (first and third column) and $\mathrm{I}_{\mathrm{V}}=100 \mathrm{ml} \mathrm{min}^{-1}$ (second and fourth column). The $\mathrm{x}$-axis is graduated in number of rotating periods. PV is integrated over each half of domain to infer the total PV embedded in the anticyclonic (dashed) and cyclonic (solid) gyre. Oscillations appear in configuration I when the forcing exceeds $\mathrm{I}_{\mathrm{V}}=50 \mathrm{ml}$ min ${ }^{-1}$, while a steady state is still reached in configuration $\mathrm{S}$ when $\mathrm{I}_{\mathrm{V}}=150 \mathrm{ml} \mathrm{min}{ }^{-1}$. From left to right: Rov $=0.13 ; 0.32 ; 0.12 ; 0.43$. Colour version online.

the two configurations is lost. Simulations in which the source is south of the sink (configuration S) are characterized by a higher level of both kinetic energy and circulation. Instantaneous maps of PVA and tracer fields (figure 4) show that the presence of these oscillations are the signature of coherent eddies. In configuration I, the central zonal jet becomes a succession of anticyclones and cyclones, with blobs of tracer being trapped in the former. The period of the oscillations shortens slightly as the flow becomes more inertial, from $232 \mathrm{~s}$ (58 inertial periods) when $\mathrm{I}_{\mathrm{V}}=50 \mathrm{ml} \mathrm{min}{ }^{-1}$, to $198 \mathrm{~s} \mathrm{(49} \mathrm{inertial} \mathrm{periods)} \mathrm{when}$

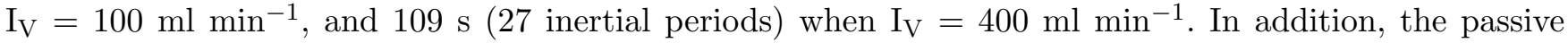
tracer now disperses all over the domain. This contrasts with the linear regime where the passive tracer is found to be mostly collocated around the source after 400 rotating periods. The presence of eddies certainly explains why the level of mean kinetic energy and the gyre's circulation is lower in this particular setup (figure 3), part of the energy input by the forcing being dissipated through the generation of eddies. In configuration $\mathrm{S}$, a quasi-stationary steady state is still reached at $\mathrm{I}_{\mathrm{V}}=200 \mathrm{ml} \mathrm{min}{ }^{-1}\left(R o_{v} \simeq 0.8\right)$. Maps of relative vorticity anomaly (not shown) actually reveal the presence of small-size-eddies embedded in the external westward jets. Nevertheless, their intensity remains much smaller than in configuration I. The gyres keep an oblong shape as they stretch towards the west, even for the most intense forcing tested

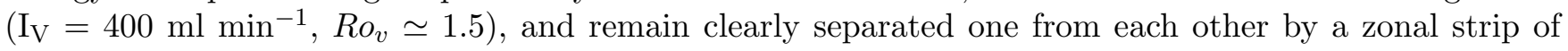
zero PVA. In addition, the passive tracer never enters the cyclonic gyre. At high forcing $\left(R o_{v} \succsim 0.8\right)$, the solution tends towards an inertial run-away type of solution (Fofonoff 1954), where inertia always dominates dissipation and any increase of the input of vorticity results in an increase of the circulation. These results are consistent with the laboratory experiments of Colin de Verdière (1977). In the latter, a transition from a laminar stable to an unstable oscillating regime also occurs above some threshold value of the forcing in configuration I, and an inertial run-away type of solution is still observed for very large values of the vorticity Rossby number $\left(R o_{v} \succsim 5\right)$ in configuration S. In the range of parameters tested numerically, we have not been able to obtain regimes with $R o_{v}$ greater than 2.0.

\section{Dynamics of the mean flow and intergyre exchanges of vorticity}

\subsection{Description of the mean flow}

The presence of two western intensified gyres separated by a zonal jet is still visible when considering the mean flow, time-averaged over more than 300 rotating periods (figure 5). The jet that separates the two gyres flows towards the east/west when the source is south (configuration S)/north (configuration I) of the sink. The external jets obviously flow in the opposite direction. They also appear to be much weaker than 

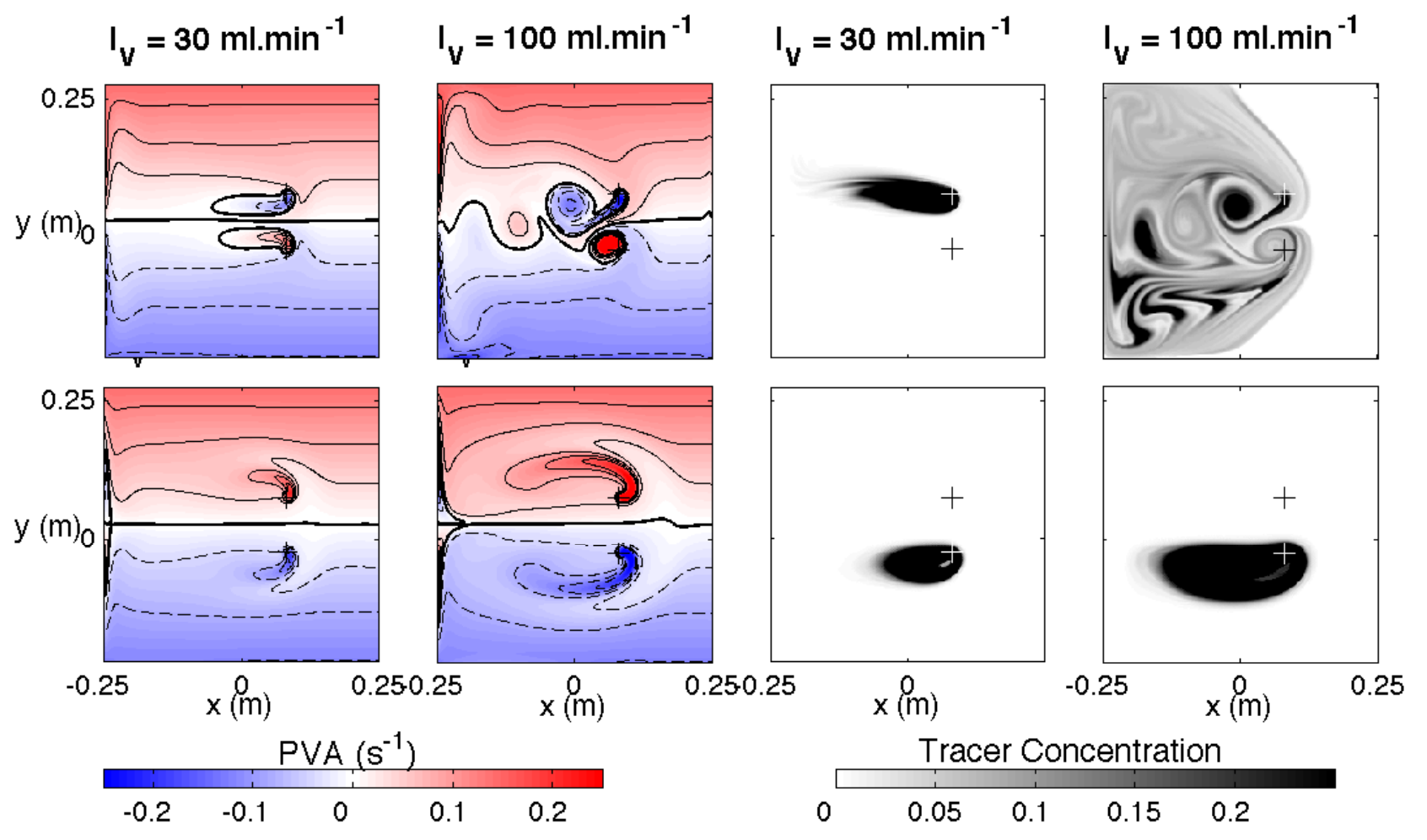

Figure 4. Instantaneous maps of PVA (first and second column) and tracer concentration (third and fourth column) at $t=400$ rotating periods in configuration I (top row) and $\mathrm{S}$ (bottom row). $\mathrm{I}_{\mathrm{V}}=30 \mathrm{ml} \mathrm{min}^{-1}$ (first and third column) and $\mathrm{I}_{\mathrm{V}}=100 \mathrm{ml} \mathrm{min}^{-1}$ (second and fourth column). $2 a=10 \mathrm{~cm}$. The input of negative/positive PVA at the source/sink appears clearly. Eddies are visible thanks to their signature in PVA in configuration I. They are generated in the zonal westward jet that separates the two gyres. In configuration S, the nonlinear solution stays stable. Note the transfer of the passive tracer from the anticyclonic to the cyclonic gyre when the source is located north (top row) of the sink. Top row, from left to right: $R o_{v}=0.13 ; 0.32 ; 0.13 ; 0.32$. Bottom row, from left to right: $R o_{v}=0.12 ; 0.43 ; 0.12$; 0.43 . Thin solid/dashed contours are for positive/negative values, and contour interval is $0.04 \mathrm{~s}^{-1}$. Colour version online.

the central jet (figure 6). Despite these similarities, there are some striking differences in the mean state, depending on whether the source is north or south of the sink. The mean circulation appears to be much more intense in simulations with a convergence of the western boundary currents, i.e in configuration $\mathrm{S}$ (figure 5, bottom row). When $\mathrm{I}_{\mathrm{V}}$ reaches $200 \mathrm{ml} \mathrm{min}{ }^{-1}$, the flow becomes inertial $\left(R o_{v} \succsim 0.8\right)$, and the recirculation of the gyres becomes twice as intense in configuration $\mathrm{S}$ than in configuration I, showing evidence of an inertial run-away type solution. In addition, within configuration S, gyres stick to an oblong shape even at a high forcing rate, with no overshooting of the western boundary currents. In configuration I, western boundary currents overshoot towards high latitudes, creating a "loop" recirculation zone (Ierley 1987, Cessi et al. 1987, Chassignet 1995). This is associated with a counter current of weaker intensity that flows opposite to the western boundary current itself, slightly offshore. The signature of these currents is shown in the x-profile of the mean western boundary currents (figure 6). The velocity is brought towards zero in a thin layer that has roughly the width of the Munk boundary layer, $\delta_{M}=1.24 \mathrm{~cm}$. This leads to strongly sheared western boundary currents with a strong signature in relative vorticity and PVA: note the band of negative/positive PVA, sitting against the western boundary, just adjacent to the cyclonic/anticyclonic gyre (figure 5).

\subsection{The mean flow vorticity dynamics}

A better understanding of the dynamics can be inferred by considering the vorticity equation (7), whose terms are shown in figure 7 for the two set-ups and a moderate inertial flow $\left(\mathrm{I}_{\mathrm{V}}=50 \mathrm{ml} \mathrm{min}{ }^{-1}, 2 a=10 \mathrm{~cm}\right.$, $R o_{v} \simeq 0.2$. In our source/sink experiments, the input of vorticity is spatially limited, and advection redistributes the negative/positive vorticity injected at the source/sink around the anticyclonic/cyclonic 


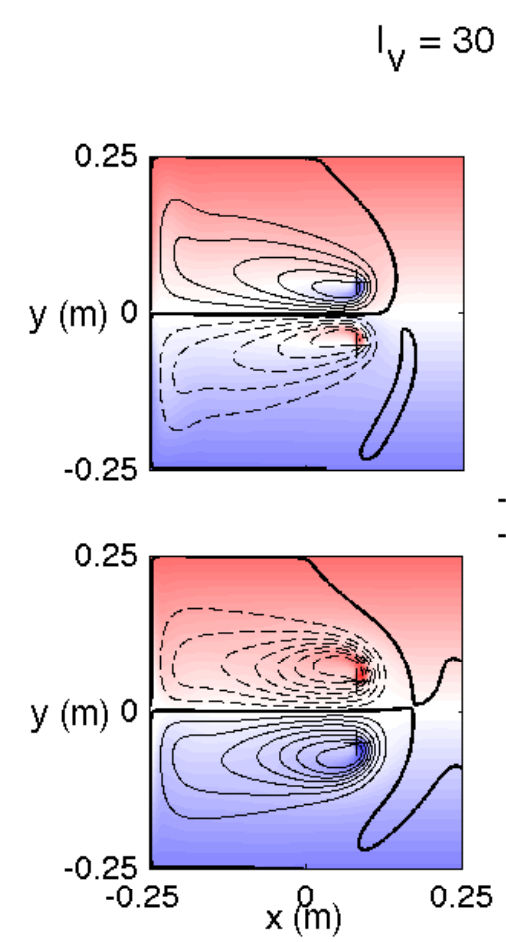

$$
\mathrm{I}_{\mathrm{v}}=30 \mathrm{ml} \cdot \mathrm{min}^{-1}
$$
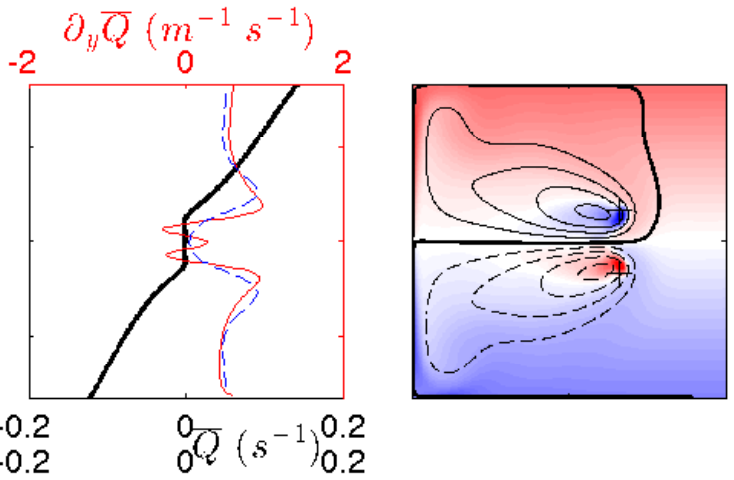

$$
\mathrm{I}_{\mathrm{V}}=100 \mathrm{ml} \cdot \mathrm{min}^{-1}
$$

\section{$-0.2$}

\section{2}
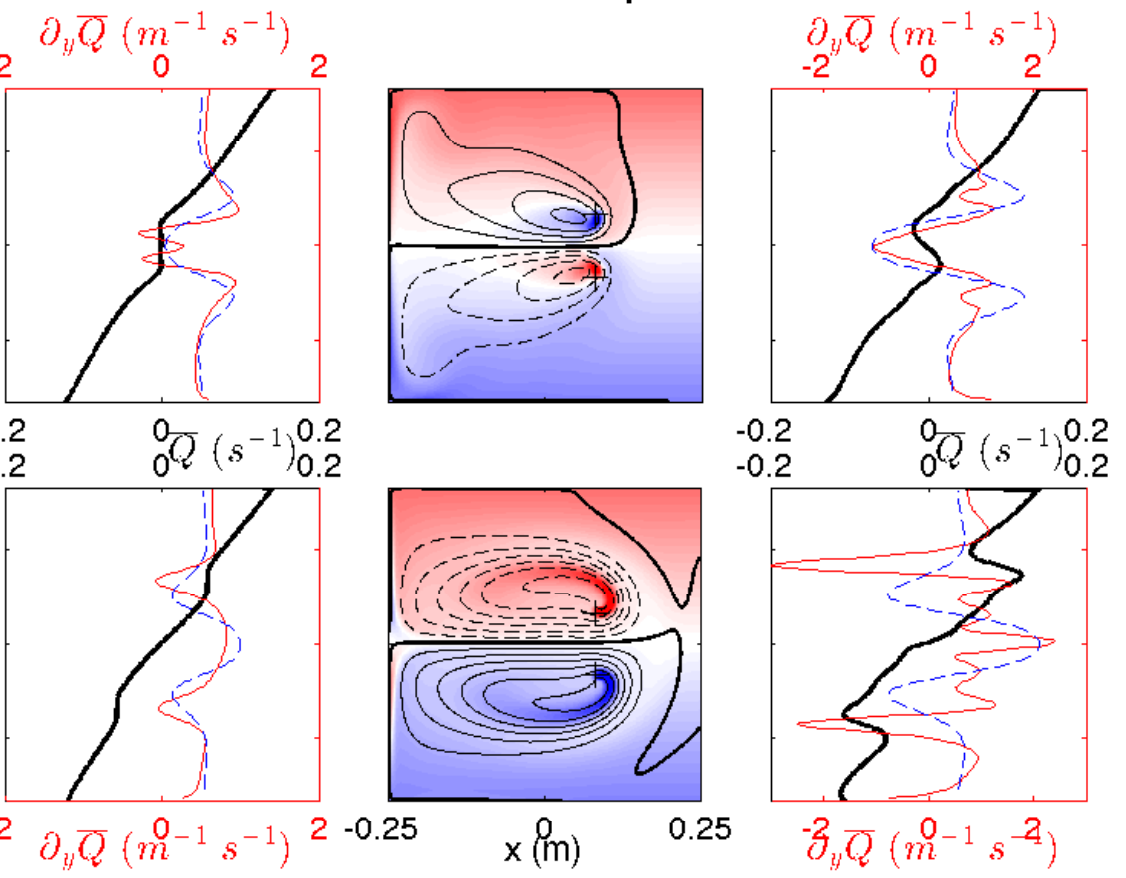

\section{PVA $\left(s^{-1}\right)$

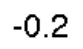 \\ $-0.1$ \\ 0.1 \\ 0.2}

Figure 5. Time-average PVA (first and third column), meridional profiles (second and fourth column) of $\bar{Q}(y)$ (black line, middle axis) and its derivative $\partial_{y} \bar{Q}(y)$ (red line, top and bottom axis) in configuration I (top row) and $\mathrm{S}$ (bottom row). $\mathrm{I}_{\mathrm{V}}=30 \mathrm{ml}^{\mathrm{min}}{ }^{-1}$ (first

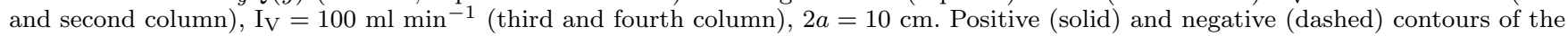
stream function $\Psi$ obtained after inverting the vorticity field are superimposed onto the PVA panels. Contour range extend in between $\pm 8 \alpha \times 10^{-5} \mathrm{~m}^{2} \mathrm{~s}^{-1}$ with a $\alpha \times 10^{-5} \mathrm{~m}^{2} \mathrm{~s}^{-1}$ interval; $\alpha=\mathrm{I}_{\mathrm{V}} / \mathrm{I}_{\text {ref }}, \mathrm{I}_{\text {ref }}=100 \mathrm{ml} \mathrm{min}{ }^{-1} \cdot \bar{Q}$ and $\partial_{y} \bar{Q}$ are averaged zonally over the interior of the domain $\left(x_{\text {source }}-0.18 \mathrm{~m}<x<x_{\text {source }}-0.08 \mathrm{~m}\right)$. Top row, from left to right: $R o_{v}=0.13 ; 0.13 ; 0.32 ; 0.32$. Bottom row, from left to right: $R o_{v}=0.12 ; 0.12 ; 0.430 .43$. Colour version online.

\section{Configuration I}

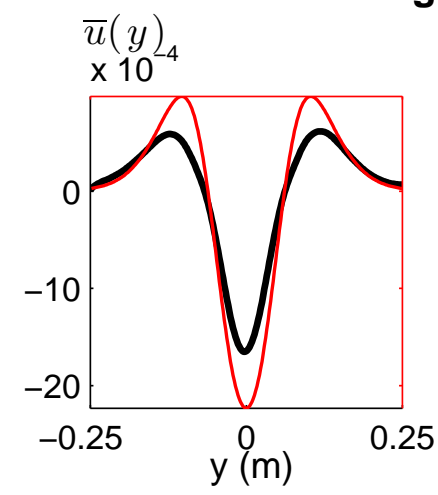

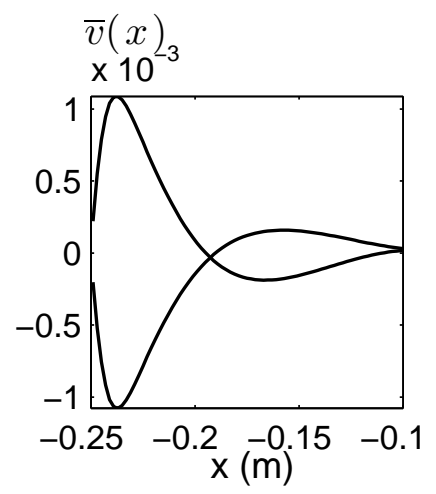

Configuration S

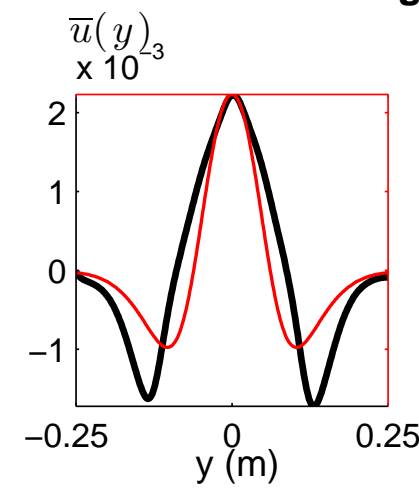

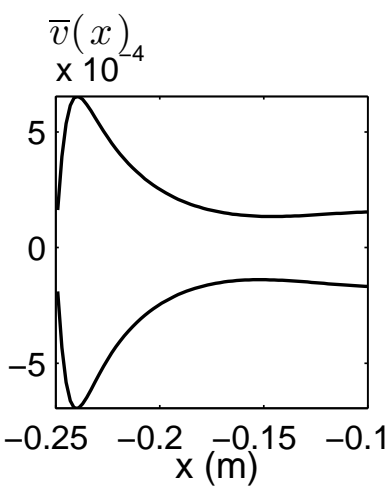

Figure 6. Time-average zonal (first and third column) and meridional (second and fourth column) velocity in configuration I (first two columns) and $\mathrm{S}$ (last two columns). $\mathrm{I}_{\mathrm{V}}=100 \mathrm{ml} \mathrm{min}{ }^{-1}, 2 a=10 \mathrm{~cm} . \bar{u}(y)$ is averaged zonally over the interior $\left(x_{\text {source }}-0.18 \mathrm{~m}<x<\right.$ $\left.x_{\text {source }}-0.08 \mathrm{~m}\right) \cdot \bar{v}(x)$ is plotted nearby the western boundary at $y= \pm 0.10 \mathrm{~m}$. The black-thick line is the result of a nonlinear numerical simulation, whereas the red-thin line is the theoretical profile deduced from equation (22). Note the presence of a counter circulation that takes place near the western boundary, slightly offshore, emphasizing the presence of a recirculation pattern in configuration I. This overshooting of the western boundary currents is not reproduced in the linear analytical solution (not shown). In the central jet, the linear analytical solution is similar to the numerical solution, even for nonlinear flows. From left to right: $R o_{v}=0.13 ; 0.32 ; 0.12 ; 0.43$. Colour version online. 
gyre. This vorticity is expelled from the region of the forcing and then redistributed over the entire gyre by the mean flow, first by the westward jets, then by the intensified western boundary currents, and finally by the eastward jets. During that path, fluid columns lose and gain some relative vorticity, partly from advection across the $f_{0} / h$ contours, in particular within the intensified western boundary currents, and on the very near eastern side of the source and sink. Nevertheless, when integrated over a defined mean streamfunction contour, the contributions of both the $\beta_{T} v$ and the mean vorticity advection term cancel (Fox-Kemper and Pedlosky 2004). Hence, the input of vorticity ends up being compensated by a combination of the viscous effects, bottom drag, and intergyre eddy vorticity fluxes (Marshall 1984, Lozier and Riser 1990, Fox-Kemper 2005). To investigate the respective contribution of bottom friction, viscous dissipation, and intergyre eddy vorticity fluxes to the PV balance of each gyre, the different terms of (7) are integrated over the shallow and deep half of the tank and the results normalized according to the input of $\mathrm{PV}$ at the source/sink $\left(f_{0} \mathrm{I}_{\mathrm{V}} / H\right)$. The $y=0$ axis is taken as the mean separation line between the anticyclonic and cyclonic gyre. Results are summarized in table 2 in percentage of the PV input. For moderate forcing $\left(R o_{v} \precsim 0.2\right)$, the input of vorticity at the source-sink is mainly balanced by the friction and viscous dissipation. Nevertheless, for intense flows in the case of divergent western boundary currents (configuration I), the intergyre eddy vorticity fluxes play a role in balancing the vorticity input. Their action takes place within the westward flowing central jet that separates the two gyres, and results in a decrease of the absolute circulation within each gyre. In this regime, the input of vorticity is mainly dissipated through intergyre eddy vorticity fluxes (not shown). The magnitude of the latter increases in magnitude (table 2) and extends westward when the flow becomes more inertial.
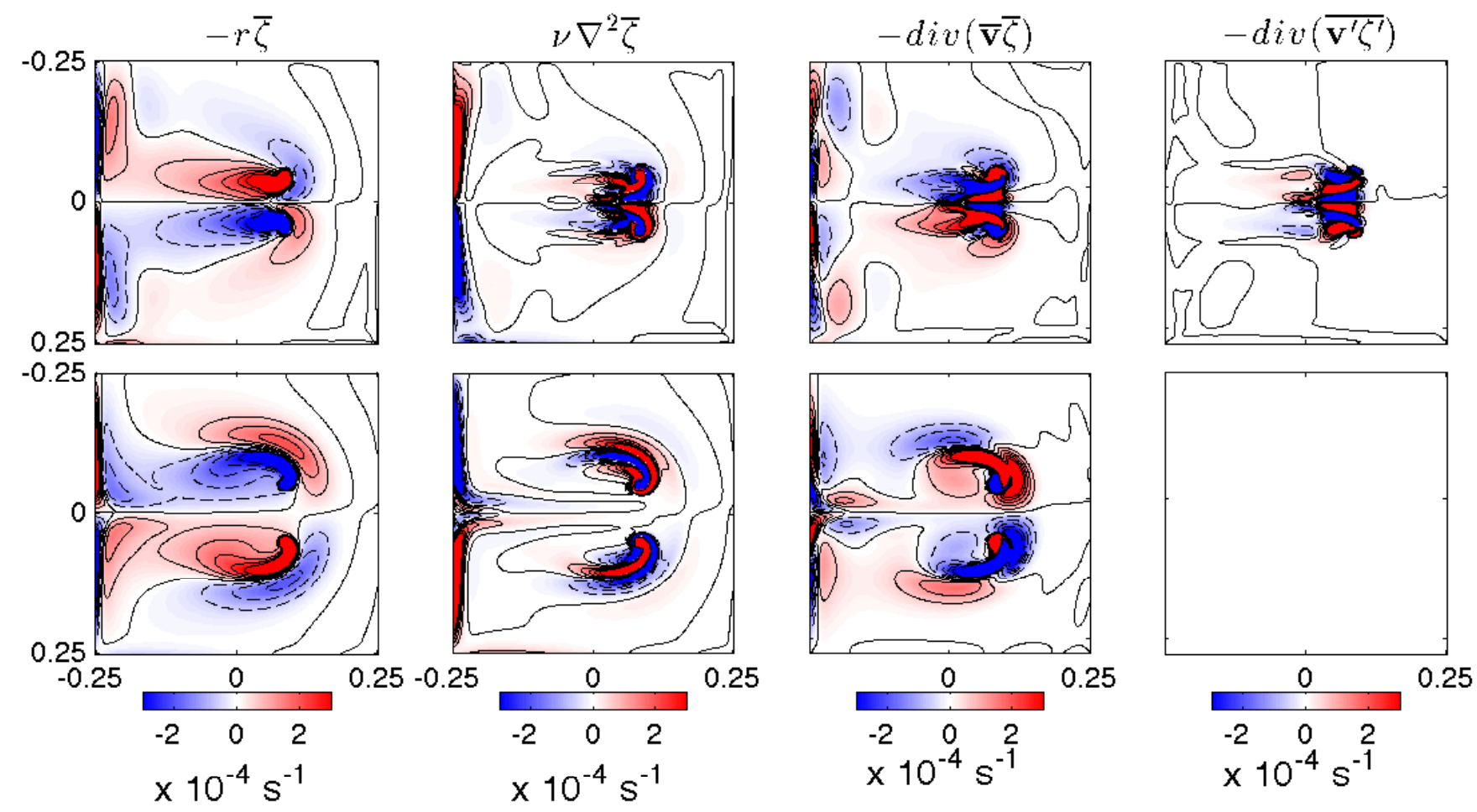

Figure 7. Time-average terms of the vorticity equation (7) in configuration I (top row) and $\mathrm{S}$ (bottom row). $\mathrm{I}_{\mathrm{V}}=50 \mathrm{ml} \mathrm{min}^{-1}$, $2 a=10 \mathrm{~cm}, R o_{v} \simeq 0.2$. Bottom friction $\left(-r \bar{\zeta}\right.$, first column), viscous dissipation $\left(\nu \nabla^{2} \bar{\zeta}\right.$, second column), divergence of the mean vorticity fluxes $\left(-\operatorname{div}(\bar{\zeta} \overline{\boldsymbol{v}})\right.$, third column), and divergence of the eddy vorticity fluxes $\left(-\operatorname{div}\left(\overline{\zeta^{\prime} \boldsymbol{v}^{\prime}}\right)\right.$, fourth column). Note the absence of eddy vorticity fluxes in the simulation in which the anticyclonic gyre is located south of the sink. Thin solid/dashed contours are for positive/negative values, and contour interval is $0.06 \mathrm{~s}^{-1}$. Colour version online. 


\section{Generation and dissipation of eddies: origin and mechanism}

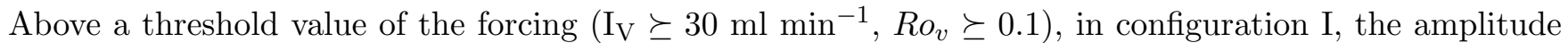
of the perturbations, here measured as the total eddy kinetic energy and enstrophy of the system, grows as the forcing increases and the eddy dynamics becomes predominant (not shown). In configuration S, perturbations also start growing, but for a much higher threshold value $\left(\mathrm{I}_{\mathrm{V}} \succsim 150 \mathrm{ml} \mathrm{min}^{-1}, R o_{v} \succsim 0.5\right)$. For the range of forcing tested, their amplitude, both in kinetic energy and enstrophy, remains an order of magnitude less than in configuration I, and the intensity of mean flow always exceeds that of the eddies.

\subsection{Origin and dissipation of eddies}
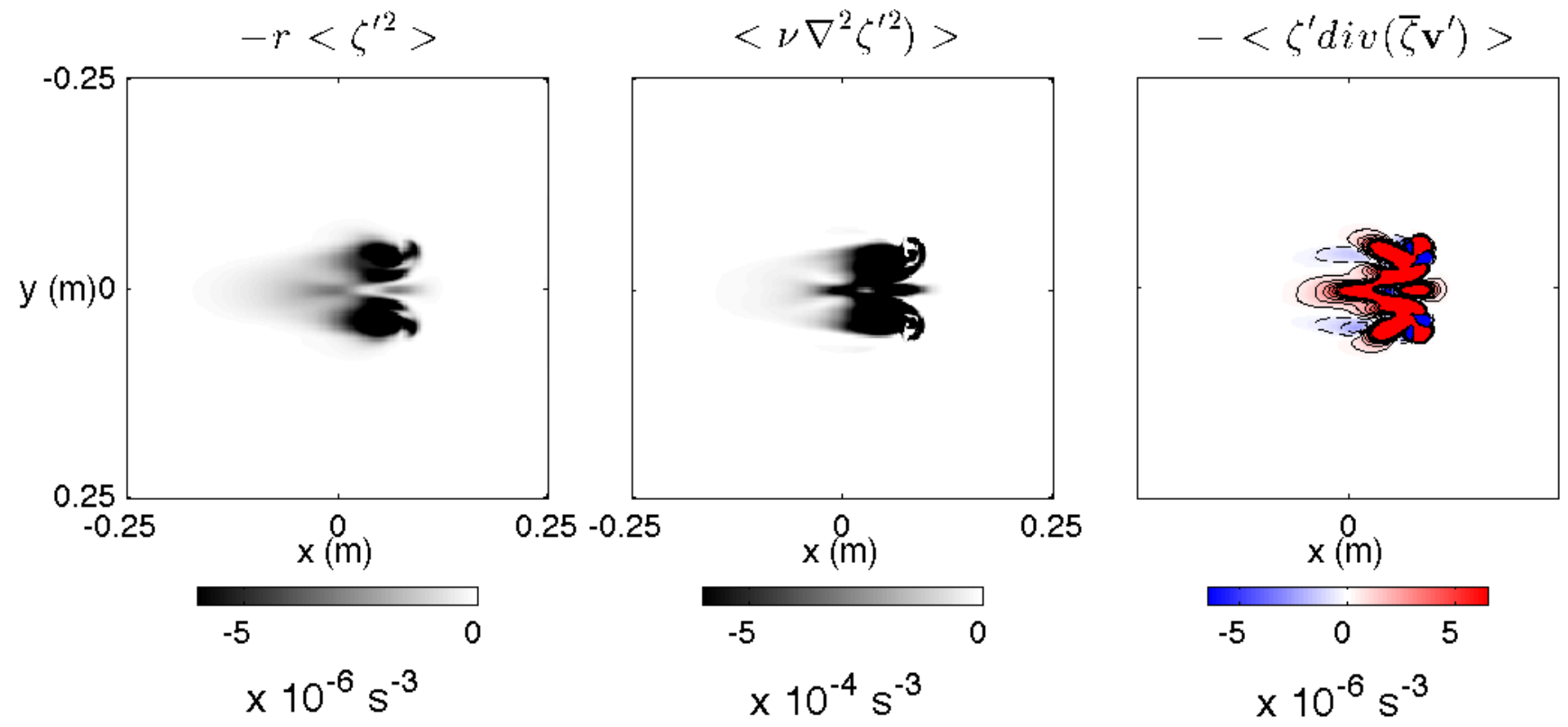

Figure 8. Time-average terms of the enstrophy equation (23) in configuration $\mathrm{I}$ for $\mathrm{I}_{\mathrm{V}}=50 \mathrm{ml} \mathrm{min}-1,2 a=10 \mathrm{~cm}, R o_{v}=0.2$. Bottom friction $\left(-r \overline{\zeta^{\prime 2}}\right.$, left), viscous dissipation $\left(\nu \nabla^{2} \overline{\zeta^{\prime 2}}\right.$, middle), divergence of the eddy vorticity fluxes across the mean-vorticity contours $\left(\operatorname{div}\left(\bar{\zeta} \boldsymbol{v}^{\prime} \zeta^{\prime}\right)\right.$, right). A time-average is performed over more than 500 rotating periods, corresponding to more than 60 oscillations in the unstable regime. Units are in $\mathrm{s}^{-3}$. In configuration $\mathrm{S}$, the enstrophy only becomes significant when $\mathrm{IV}_{\mathrm{V}}$ exceeds $150 \mathrm{ml} \mathrm{min}^{-1}$. Smallamplitude eddies then start to appear in the external jets. Thin solid/dashed contours are for positive/negative values. Contour interval is $1.0 \mathrm{~s}^{-3}$ in the range $[-6.5,6.5] \mathrm{s}^{-3}$. Colour version online.

We first try to locate in space the growth of the instability. The latter may either come from intrinsic instabilities of the zonal jets and/or the western boundary currents or from the forcing itself. Because the generated eddies have a strong signature in relative vorticity, their growth and dissipation can be inferred from the equation of evolution of enstrophy, i.e. $\zeta^{\prime 2}=(\zeta-\bar{\zeta})^{2}$ (Rhines and Holland 1979, Rhines 1979, Marshall 1984, Lozier and Riser 1990):

$$
\begin{aligned}
& \begin{array}{cccc}
<\frac{\partial \zeta^{\prime 2}}{\partial t}> & +\frac{1}{2}<\overline{\boldsymbol{v}} \cdot \boldsymbol{\nabla} \zeta^{\prime 2}> & +<\zeta^{\prime} \boldsymbol{v}^{\prime} \cdot \nabla \bar{\zeta}>+<\zeta^{\prime} \nabla \cdot\left(\boldsymbol{v}^{\prime} \zeta^{\prime}\right)>=-<\zeta^{\prime 2} \boldsymbol{\nabla} \cdot \overline{\boldsymbol{v}}>\ldots \\
T 1 & T 2 & T 3 & T 5
\end{array} \\
& -\left\langle\zeta^{\prime}\left(f_{0}+\bar{\zeta}\right) \nabla \cdot v^{\prime}>\quad-<r \zeta^{\prime 2}>\quad+<\nu \zeta^{\prime} \nabla^{2} \zeta^{\prime}>\right. \\
& \begin{array}{lll}
T 6 & T 7 & T 8
\end{array}
\end{aligned}
$$

Here, $\bar{\zeta}$ and $\overline{\boldsymbol{v}}$ stand for the vorticity and velocity vector of the mean flow, $\zeta^{\prime}$ and $\boldsymbol{v}^{\prime}$ are the relative vorticity and velocity perturbations. We apply a time-average operator over 60 periods of oscillations (hereafter represented by the symbol $\langle\cdot\rangle$ ), so that the first order perturbations terms cancel. The different terms 
of (23) all contribute locally to the variation of enstrophy, either by redistributing spatially, dissipating, or increasing the amplitude of the perturbations themselves. The spatial structure of the dominant term for the generation of enstrophy, $T 3$, is linked to the eddy flux of vorticity across the contours of mean vorticity. $T 3$ is found to be responsible for the growth of enstrophy within the zonal westward jets above a threshold value of the forcing (figure 8). That value varies whether the source is located north or south of the sink. In configuration I, i.e. in the simulations with the westward jet confined in between the two gyres, this threshold value is in between $\mathrm{I}_{\mathrm{V}}=30 \mathrm{ml} \mathrm{min}{ }^{-1}\left(R o_{v}=0.1\right)$ and $\mathrm{I}_{\mathrm{V}}=50 \mathrm{ml} \mathrm{min}^{-1}\left(R o_{v}=0.2\right)$. $T 3$ is then found to increase in magnitude as $\mathrm{I}_{\mathrm{V}}$ increases. In configuration $\mathrm{S}$, the threshold value is about five times higher, and the growth rate several order of magnitude weaker. $T 7$ and $T 8$ are linked to the dissipation of the perturbations by bottom friction and viscosity. As expected, $T 7$ and $T 8$ behave as a sink of enstrophy. We find that perturbations are mostly dissipated in the central zonal jet and do not reach the western boundary (figure 8).

\subsection{Sensitivity to the source/sink distance: effect on barotropic instability}

The above analysis suggests that the growth of perturbations in our double-gyre system is linked to the instability of the westward zonal jets. A zonal barotropic jet can only be unstable if the PV gradient vanishes somewhere (Kuo 1949, Pedlosky 1987):

$$
\partial_{y}\left(-\partial_{y} u(y)+\beta y\right)=0, \text { somewhere in the flow. }
$$

Sensitivity experiments varying the intensity of the forcing (figure 5) and the distance between the source and sink (figure 9) reveal that the growth of eddies is always associated with the fulfilment of equation (24) in the westward central jet, whereas the jet remains stable when the latter criteria is not satisfied. Increasing the intensity of the source-sink dipole, either by increasing $\mathrm{I}_{\mathrm{V}}$ or decreasing $2 a$ amplifies the magnitude of the PV gradient associated with the jet confined in between the two gyres, and therefore facilitates the fulfilment of equation (24), provided that the sign of this gradient is opposite to $\beta$, i.e in the case of westward zonal jets. In configuration I, when the transition from a stable to a unstable oscillating regime occurs for moderate inertial flows, the fulfilment of the Kuo criteria by the linear analytical solution provides a good estimate of the bifurcation. In configuration S, despite the criteria is fulfilled as soon as $\mathrm{I}_{\mathrm{V}} \simeq 75 \mathrm{ml} \mathrm{min}^{-1}$, instabilities in the external jets are only seen for $\mathrm{I}_{\mathrm{V}}$ above $150 \mathrm{ml} \mathrm{min}{ }^{-1}$. The position and PV structure of the jets in the analytical and numerical solution then differ substantially. Note that we have also found some PV gradient reversals within the western boundary currents. Nevertheless, this reversal has never led to the generation of energetic eddies.

\subsection{Linear stability analysis}

In order to investigate the type of bifurcation that occurs when the circulation shifts from a steady to an oscillatory regime as the nonlinear parameter $R o_{v}$ is increased, we perform a linear stability analysis (LSA) of the mean states. Equations (1) are first linearised for perturbations $X^{\prime}$ from a steady or mean state $\bar{X}\left(X^{\prime}=X-\bar{X}\right)$, where $X$ is composed of the state variables $\left(h_{i}, \boldsymbol{v}_{i}\right)$ of the system, $i$ being a spatial index. The LSA consists in computing the eigenvalues $\omega$ and eigenvectors of this linear system:

$$
\partial_{t} X^{\prime}=J X^{\prime}=\omega X^{\prime}
$$

where $J$ is the Jacobian matrix depending on the mean state $\bar{X}$. The latter is computed by time-averaging the numerical solution over $5000 \mathrm{~s}$, which corresponds roughly to 60 oscillation periods in the case of the oscillating regime (figure 3). We only look for the 30 leading eigenvalues in terms of growth rate $\omega_{r}$, i.e the real part of $\omega\left[\omega_{r}=\Re(\omega)\right]$. In fact, these eigenvalues capture the large scale eigenvectors that are relevant for our problem. The remaining part of the spectrum has finer scale. Hence, it is strongly damped through the scale-selective viscosity. We use an iterative Arnoldi method in Krylov subspace for the computation of 

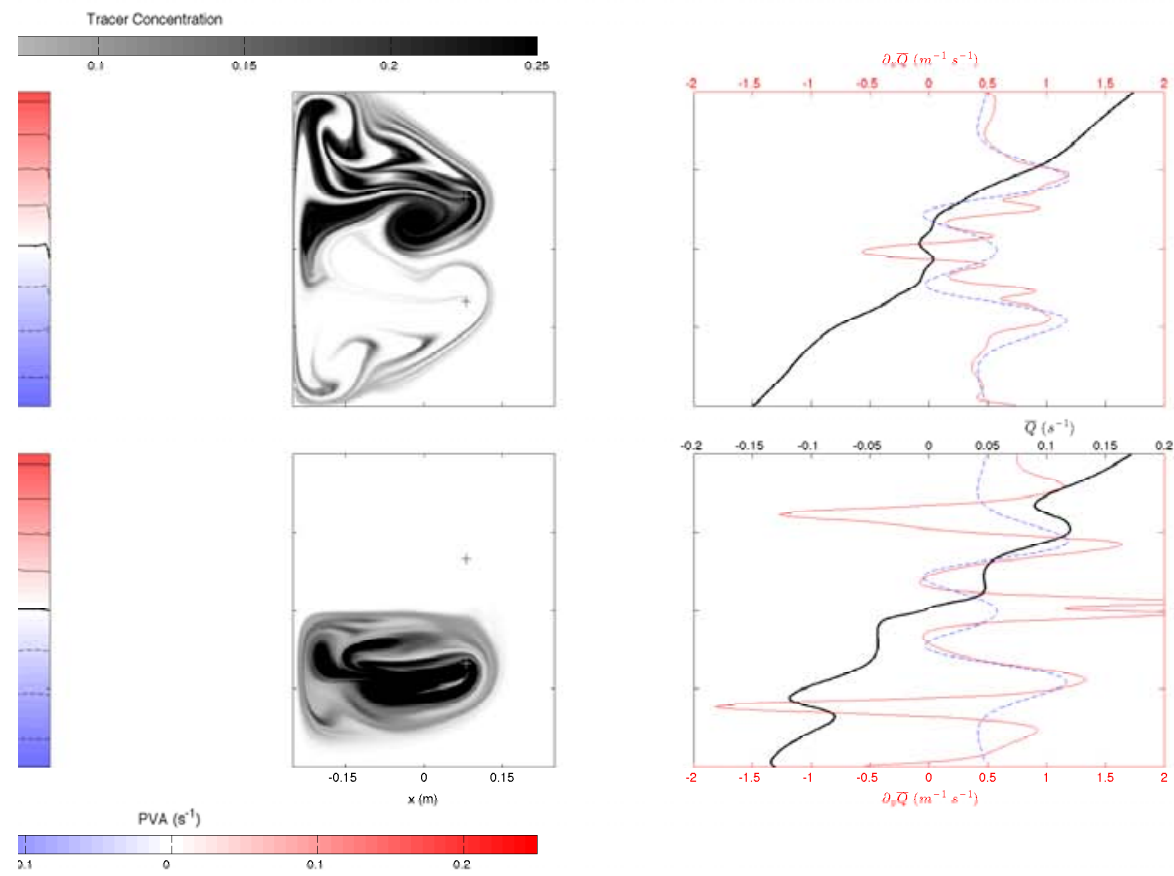

Figure 9. Instantaneous maps ( $t=400$ rotating periods) of PVA (left), tracer distribution (middle), and profiles of $\bar{Q}(y)$ and its derivative (right) in configuration I (top row) and S (bottom row) for $\mathrm{I}_{\mathrm{V}}=100 \mathrm{ml} \mathrm{min}^{-1}, 2 a=20 \mathrm{~cm} . \bar{Q}(y)$ (black thick) and $\partial_{y} \bar{Q}(y)$ (red thin) are averaged zonally over the basin interior $x_{\text {source }}-0.18 \mathrm{~m}<x<x_{\text {source }}-0.08 \mathrm{~m}$. The dashed-blue line represents $\partial_{y} \bar{Q}(y)$ derived from the analytical solution. $R o_{v}=0.08$ in configuration I (top row) and $R o_{v}=0.06$ in configuration S (bottom row). Thin solid/dashed contours are for positive/negative values, and contour interval is $0.04 \mathrm{~s}^{-1}$. Colour version online.

the eigenvalues and eigenvectors as provided in ARPACK (Lehoucq et al. 1998). Given the dimension of $X$ $(\sim 250 \times 250 \times 3)$, computing the Jacobian matrix explicitly would require too much memory. Instead, we integrate the tangent linear model for a given time $\tau$. Several tests have been performed and have shown robust results for values of $\tau$ of the order of $1 \mathrm{~s}$. This time is large enough to filter out the gravity waves, and short enough to resolve accurately the topographic Rossby waves that are responsible for the observed oscillations. ARPACK provides the eigenvalues $(\exp (\omega \tau))$ and eigenvectors of the so-called propagator $M(\tau)$, from which we compute the Jacobian eigenvalues $\omega$ :

$$
X^{\prime}(t=\tau)=M(\tau) X^{\prime}(t=0)=\mathrm{e}^{J \tau} X^{\prime}(t=0)=\mathrm{e}^{\omega \tau} X^{\prime}(t=0)
$$

The spectrum of the eigenvalues $\omega=\omega_{r} \pm \mathrm{i} \omega_{i}$ is computed for increasing values of the forcing in configuration I and $2 a=10 \mathrm{~cm}$ (figure 10, top left). The results agree with the integrations of the nonlinear model: i) all eigenvalues are damped (negative real part) when the forcing is below a threshold value, here $\mathrm{I}_{\mathrm{V} c}=47 \mathrm{ml} \mathrm{min}^{-1}\left(R o_{v} \simeq 0.2\right)$; ii) there is a single eigenvalue with a positive real part that corresponds to an oscillation of period $200 \mathrm{~s}$ ( $\simeq 50$ inertial periods), similar to the unstable mode found in the nonlinear integration. We isolate that mode and plot the variation of its eigenvalue for different amplitudes of the forcing (figure 10, bottom left) The growth rate switches from negative (damped perturbation) to positive (growing perturbation) value when $\mathrm{I}_{\mathrm{V}}$ reaches a threshold value. The period of the oscillation $\left(2 \pi / \omega_{i}\right)$ shortens slightly as $I_{V}$ is increased from 45 to $400 \mathrm{ml} \mathrm{min}^{-1}$. The spatial structure of the eigenvectors (figure 10, right) corresponds to a Rossby wave travelling westward. The latter is intensified in the westward central jet, i.e. the region previously identified as barotropically unstable. We have also applied the LSA to mean states resulting from configuration S. Results (not shown) have confirmed a threshold value of the forcing around $\mathrm{I}_{\mathrm{V} c}=150 \mathrm{ml} \mathrm{min}{ }^{-1}\left(R o_{v} \simeq 0.5\right)$ for the growth of an unstable mode, and a perturbation intensified in the external westward jets.

The LSA allows us to characterize the bifurcation that occurs when the forcing is increased. Near the threshold value of the regime transition, the period of oscillation of the unstable mode remains constant while its growth rate switches from a negative to a positive value (figure 10, bottom left). For a large 


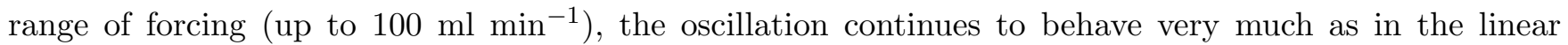
regime, i.e. with a period and spatial structure very similar to the unstable linear eigenvector. Only the finite amplitude of the oscillation makes it a nonlinear process. This behaviour suggests that the regime transition is linked to a supercritical Hopf (pitchfork) bifurcation. For a simple system obeying a Hopf bifurcation, near the transition regime, the square amplitude of the perturbation should grow linearly with the forcing anomaly $\mathrm{I}_{V}-\mathrm{I}_{V c}$ (Strogatz 1994). We have computed the time-average volume eddy kinetic energy $\left(\overline{\mathrm{EKE}}_{\mathrm{V}}\right)$ and enstrophy $\left({\overline{\zeta^{\prime 2}}}_{V}\right)$ near the transition regime in our numerical experiments, for $\mathrm{I}_{\mathrm{V}}$ in between 30 and $50 \mathrm{ml} \mathrm{min}^{-1}$ (not shown). The threshold is slightly lower than the one given by the LSA $\left(\mathrm{I}_{\mathrm{V} c} \simeq 32 \mathrm{ml} \mathrm{min}^{-1}\right)$, and the amplitude of the perturbation increases slower than the one predicted by the theory. These differences could come from the fact that we are dealing with a dynamical system with more than just one degree of freedom.

The LSA provides an ideal framework where the mean state and the perturbations are perfectly separated: We take advantage of knowing exactly the spatial structure of the most unstable perturbation to rationalize its growth and dissipation by performing an energy budget. Because the flow considered in our simulations is characterized by small Froude numbers and weak free surface deviations, we only focus on the volume integrated kinetic energy $\mathrm{KE}_{V}$ : the latter is found to be two order of magnitude larger than the potential energy. After performing a Reynolds decomposition into mean and perturbation, the expression for $\mathrm{KE}_{V}$ writes as follows:

$$
\begin{aligned}
\mathrm{KE}_{V} & =\int_{\mathcal{D}} h\left(\frac{u^{2}+v^{2}}{2}\right) \mathrm{d} A=\int_{\mathcal{D}}\left(\bar{h}+h^{\prime}\right)\left(\frac{\left(\bar{u}+u^{\prime}\right)^{2}+\left(\bar{v}+v^{\prime}\right)^{2}}{2}\right) \mathrm{d} A \\
& =\int_{\mathcal{D}} \bar{h}\left(\frac{\bar{u}^{2}+\bar{v}^{2}}{2}\right) \mathrm{d} A+\int_{\mathcal{D}} \bar{h}\left(\frac{u^{\prime 2}+v^{\prime 2}}{2}\right) \mathrm{d} A+\int_{\mathcal{D}} h^{\prime}\left(\bar{u} u^{\prime}+\bar{v} v^{\prime}\right) \mathrm{d} A .
\end{aligned}
$$

Equation (28) that suggests the volume integrated eddy kinetic energy $\mathrm{EKE}_{V}$ cannot be restricted to the second term. Nevertheless, practically, computing these terms for the eigenvectors shows that the third term is more than $10^{6}$ times smaller. Therefore, we define the depth integrated eddy kinetic energy as:

$$
\mathrm{EKE}=\bar{h}\left(\frac{u^{\prime 2}+v^{\prime 2}}{2}\right)
$$

In the weakly nonlinear regime, the latter is linked to the growth of the most unstable mode. After some algebra, its equation of evolution reads:

$$
<\partial_{t} \mathrm{EKE}>=<\mathrm{EKE}_{\nabla}>+<\mathrm{EKE}_{r}>+<\mathrm{EKE}_{\nu}>,
$$

where $\mathrm{EKE}_{\nabla}, \mathrm{EKE}_{r}$ and $\mathrm{EKE}_{\nu}$ are the respective contributions of advection, friction, and viscous dissipation:

$$
\begin{aligned}
& \mathrm{EKE}_{\nabla}=-\bar{h} \overline{\boldsymbol{v}} \cdot \boldsymbol{\nabla}\left(\frac{u^{\prime 2}+v^{\prime 2}}{2}\right)-\bar{h} \boldsymbol{v}^{\prime} \cdot \nabla\left(\bar{u} u^{\prime}+\bar{v} v^{\prime}\right) \\
& \mathrm{EKE}_{r}=-2 r \bar{h}\left(\frac{u^{\prime 2}+v^{\prime 2}}{2}\right) \\
& \mathrm{EKE}_{\nu}=\nu \bar{h}\left(u^{\prime} \nabla^{2} u^{\prime}+v^{\prime} \nabla^{2} v^{\prime}\right) .
\end{aligned}
$$

Note that we show only the quadratic terms that have a significant contribution to the global average (all the others have also been computed but have contributions $10^{3}$ times smaller than the ones shown). We 
use the notation $\langle\cdot\rangle$ for time-average over one period of oscillation $T=2 \pi / \omega_{i}$ :

$$
<\cdot>=\frac{1}{T} \int_{t-\frac{T}{2}}^{t+\frac{T}{2}} \cdot\left(t^{\prime}\right) \mathrm{d} t^{\prime} .
$$

For the oscillating modes found through the LSA, eigenvalues come as a pair of complex conjugates $\omega=\omega_{r} \pm \mathrm{i} \omega_{i}$, as well as the eigenvectors $X^{\prime}=X_{r} \pm \mathrm{i} X_{i}$, and the associated solution in the real space reads:

$$
X^{\prime}(t)=\Re\left\{\left(X_{r}+\mathrm{i} X_{i}\right) \exp \left[\left(\omega_{r}+\mathrm{i} \omega_{i}\right) t\right]\right\}=\left[X_{r} \cos \left(\omega_{i} t\right)-X_{i} \sin \left(\omega_{i} t\right)\right] \exp \left(\omega_{r} t\right) .
$$

We assume that the amplitude $\left(\exp \left(\omega_{r} t\right)\right)$ varies on a time scale $\tau=1 / \omega_{r}$ much larger than $T$, so that the first order terms of the oscillating perturbation cancel and the quadratic terms can be estimated as follows:

$$
<X^{\prime} Y^{\prime}>(t)=\frac{1}{T} \int_{t-\frac{T}{2}}^{t+\frac{T}{2}} X^{\prime}\left(t^{\prime}\right) Y^{\prime}\left(t^{\prime}\right) \mathrm{d} t^{\prime} \simeq \frac{X_{r} Y_{r}+X_{i} Y_{i}}{2} \exp \left(2 \omega_{r} t\right) .
$$

The spatial structure of the leading terms of (30) is shown in figure 11 for a forcing of $+100 \mathrm{ml} \mathrm{min}^{-1}$ in configuration I. The region where the nonlinear source term is mostly positive, westward of the source and sink, clearly agrees with the barotropic instability criteria and the enstrophy budget performed earlier on the nonlinear oscillation. In addition, after scaling the leading terms in the perturbations kinetic energy budget by twice the total kinetic energy, and summing them all together, we are able to retrieve the exact growth rate found by the LSA:

$$
\frac{<\overline{\partial_{t} \mathrm{EKE}}>}{2<\overline{\mathrm{EKE}}>}=\frac{<\overline{\mathrm{EKE}_{\nabla}}+\overline{\mathrm{EKE}_{\nu}}+\overline{\mathrm{EKE}_{r}}>}{2<\overline{\mathrm{EKE}}>}=\omega_{r}
$$

The contribution from the nonlinear term is clearly the source term for the oscillation: $+8.86 \times 10^{-3} \mathrm{~s}^{-1}$, balanced by bottom friction: $-6.00 \times 10^{-3} \mathrm{~s}^{-1}$ and viscosity: $-2.15 \times 10^{-3} \mathrm{~s}^{-1}$; their sum gives $+7.118 \times$ $10^{-4} \mathrm{~s}^{-1}$, very close to $\omega_{r}=+7.113 \times 10^{-4}$. In addition, we have computed the eddy PV fluxes associated with the unstable mode for mean states under various forcing amplitude: as expected, their structure is exactly the same as the ones computed in the nonlinear experiments (not shown).

\section{$7 \quad$ Summary and discussion}

This work revisits theoretically and numerically the laboratory experiments of Colin de Verdière (1977), in which a mass source-sink dipole is used to force a double zonally-elongated gyre on a $\beta$-plane with a western boundary. For the first time, a linear analytical solution of a simplified double-gyre system with intensified western boundary currents closing the circulation is derived. Its robustness to the degree of nonlinearity of the flow is tested numerically using the MICOM shallow-water model. The nondimensional parameter $R o_{v}=U /\left(4 \beta a^{2}\right.$ ) (with $U$ the maximum zonal velocity of the flow and $2 a$ the distance between the source and the sink) is used as a measure of the nonlinearity of the flow. Sensitivity experiments to the relative position of the source and sink, and to the intensity of the dipole, either by varying the amplitude of the mass injection and pumping or the source-sink distance, confirm the findings of Colin de Verdière (1977): i) when the source is north of the sink (configuration I), the flow becomes unstable as soon as $R o_{v}$ reaches 0.1 ; ii) when the source is south of the sink (configuration S), the flow remains stable until much larger values of $R o_{v}(\simeq 0.5)$, and the flow then begins to share some of the characteristics of a purely inertial flow.

We analyse the stability properties of the flow by considering the point of view of Kuo (1949)'s instability criteria, enstrophy budgets, and linear stability analysis (LSA). All three methods converge to attribute the asymmetry observed in the stability of the two configurations to the growth of an unstable mode 

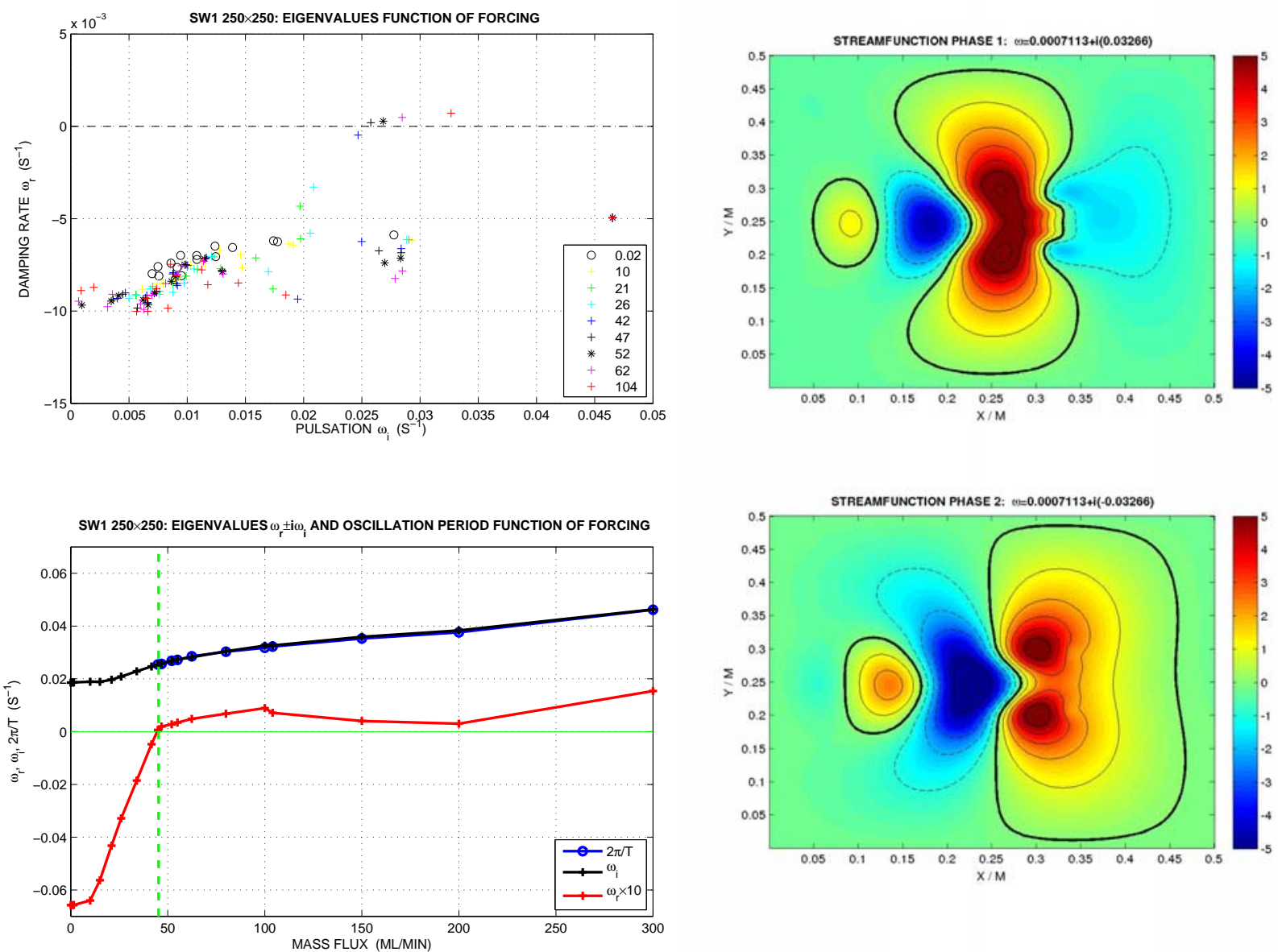

Figure 10. Linear stability analysis. a) Spectrum of the leading eigenvalues $\omega=\omega_{r} \pm i \omega_{i}$ in configuration I for increasing values of $I_{V}$ and $2 a=10 \mathrm{~cm}$ (top left). The mean flow promotes one single oscillatory eigenvector, whose growth rate increases from negative to positive values, while its period $\left(T=2 \pi / \omega_{i}\right)$ decreases from $300 \mathrm{~s}$ (no forcing), to $235 \mathrm{~s}$ (at the bifurcation) and $200 \mathrm{~s}\left(\right.$ for $\mathrm{I}_{\mathrm{V}}=100 \mathrm{ml} \mathrm{min}{ }^{-1}$ ). b) Leading eigenvalue (real and imaginary parts) and nonlinear oscillation pulsation as a function of $\mathrm{I}_{\mathrm{V}}$ in configuration I (bottom left). We compare the imaginary part of the fastest growing mode $\left(\omega_{i}\right.$, black +$)$ with the nonlinear oscillation pulsation $(2 \pi / T$, blue o) only known after the bifurcation (green dashed). c) Eigenvector of the fastest growing mode in configuration I for $\mathrm{IV}_{\mathrm{V}}=100 \mathrm{ml} \mathrm{min}^{-1}$ and $2 a=10 \mathrm{~cm}$ (right). The oscillation period is $192 \mathrm{~s}$ and the growth rate $1406 \mathrm{~s}^{-1}$. Thick line: zero value; dashed lines: negative values; solid lines: positive value (amplitude is arbitrary). The two phases of the layer thickness are separated by a quarter period and evolve as: PHASE $1 \rightarrow-$ PHASE $2 \rightarrow$ - PHASE $1 \rightarrow$ PHASE $2 \ldots$ The topographic Rossby wave propagates westward and is amplified by the barotropic instability that occurs within the westward jet, in between the source and sink. Colour version online.

intensified in the westward jets. Indeed, the Kuo (1949)'s criteria, based on the necessity to have an inversion of the PV gradient somewhere in the flow, can only be met within the westward jets whose relative vorticity gradient opposes the ambient topographic PV gradient, $\beta_{T}$. It is satisfied for less intense forcing in configuration I than in configuration $\mathrm{S}$, because the central westward jet is more sheared than the external ones. Budgets of eddy kinetic energy (computed from the unstable mode given by the LSA) and enstrophy (computed from the numerical solution) show that the perturbations are damped in the westward jets before they reach the western boundary.

The novelty of the experimental setup is that the fluid is forced locally as compared to wind-driven gyres where the forcing is distributed over the entire basin. Indeed, the analytical solution is the Green function of the barotropic $\beta$-plane wind-driven gyre problem with a western boundary. The circulation which is free almost everywhere, results from advection of vorticity injected solely at the source and sink, and compensation by advection of fluid columns across the ambient PV gradient. For weakly inertial flows, the balance of vorticity is achieved by Ekman friction and viscous dissipation. When the flow becomes more inertial, eddies are generated through barotropic instability of the westward jets. They provide a route towards dissipation of the input of vorticity before it enters the western boundary layers. In addition, in configuration I, they contribute significantly to the irreversible exchange of water masses between the 

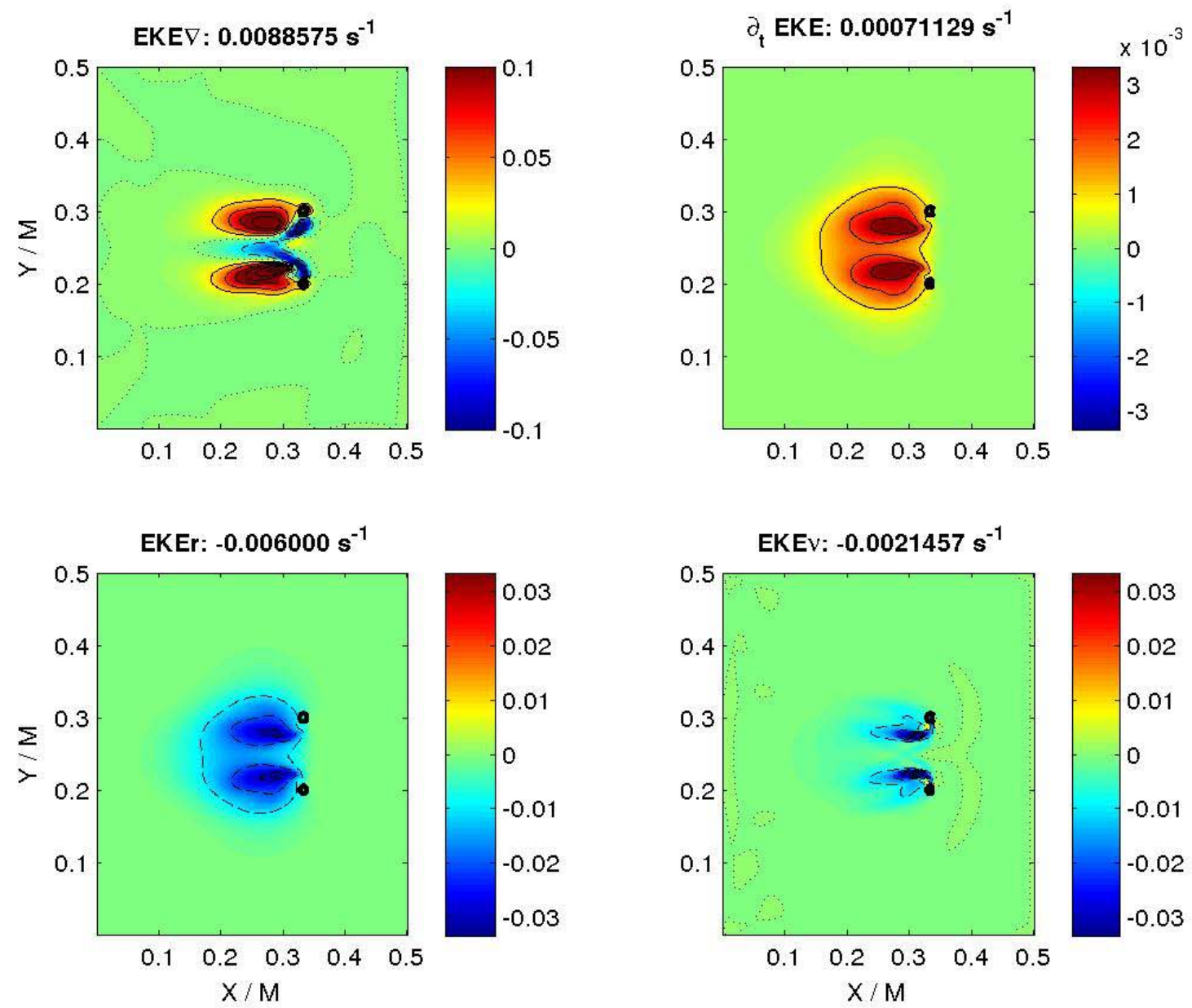

Figure 11. Energy budget of the leading eigenvector for the simulation corresponding to $\mathrm{I}_{\mathrm{V}}=100 \mathrm{ml} \mathrm{min}^{-1}$ and $2 a=10 \mathrm{~cm}$ in configuration I. Nonlinear eddy kinetic energy source term $\mathrm{EKE}_{\nabla}$ (top left); dissipation by bottom friction $\mathrm{EKE}_{r}$ (bottom left); dissipation by viscosity $\mathrm{EKE}_{\nu}$ (bottom right); eddy kinetic energy growth rate (top right). Contributions to the EKE budget are scaled by twice the total EKE, such that they sum up to the growth rate of the unstable mode. Note that the color scale varies according to the panel. The black circles show the source and sink location. Thin solid/dashed/dotted contours are for positive/negative/zero values. Contour interval is $0.03 \mathrm{~s}^{-1}$ for $\mathrm{EKE}_{\nabla}$ (top left), $0.001 \mathrm{~s}^{-1}$ for $\partial_{t} \mathrm{EKE}$ (top right), $0.01 \mathrm{~s}^{-1}$ for $\mathrm{EKE}_{r}$ (bottom left) and $\mathrm{EKE}_{\nu}$ (bottom right). Colour version online.

anticyclonic and cyclonic gyres (Lozier and Riser 1990, Fox-Kemper 2005).

One puzzling result from our experiments is the absence of eddies and meanders in the eastward jets, in particular in the regions of separation of the western boundary currents. In our simulations, in configuration $\mathrm{S}$, the tongues of $\mathrm{PV}$ that emerge from the western boundary mix together to create a region of homogeneous PV equal to zero, which prevents the possibility to have PV gradients reversals in the eastward jet. This mechanism contrasts with the homogenization of PV occurring all around each gyre due to shear enhanced mixing, as described in Rhines and Young (1982). Here, there is homogenization of the PV coming from the anticyclonic and cyclonic reservoir at the convergence of the western boundary currents. The strict anti-symmetry in our forcing probably explains why tongues of PV advected in the eastward jets exactly compensate each other (Moro 1990, Cessi 1991, Haidvogel et al. 1992). Ierley (1987) and Ierley and Young (1991) also emphasize the possibility for viscous instabilities to take place in the viscous western boundary layer provided the Reynolds number was above some threshold value. Those instabilities are hypothesized to trigger the generation of mesoscale eddies at the separation of the western boundary currents (Chassignet 1995, Verron and Le Provost 1991). However, the presence of bottom friction in our simulations and the lack of resolution in a viscous sub-layer $\left(l=\sqrt{\left.\delta_{I} \nu\right) / U} \simeq 4 \mathrm{~mm}\right.$ for $\mathrm{I}_{\mathrm{V}}=100 \mathrm{ml} \mathrm{min}^{-1}$ and $2 a=10 \mathrm{~cm}$ ) (Pedlosky 1987) may explain why we never find the growth of eddies in the western 
boundary currents. The presence of enhanced friction in our simulations may also explain why, in our configuration $\mathrm{S}\left(R o_{v}^{\max } \simeq 2\right)$, we do not find such intense inertial flows such as the ones observed in Colin de Verdière $(1977)\left[R o_{v}^{\max } \simeq 5\right]$. Because in our simulations, increasing $\mathrm{I}_{\mathrm{V}}$ above $400 \mathrm{ml} \mathrm{min}{ }^{-1}\left(R o_{v}>1.4\right)$ leads to the interaction of the double-gyre with the lateral boundaries, and sometimes to a numerical instability near these boundaries, we have not been able to conduct such experiments. That interaction could enhance the meridional shear of the external jets and favour instability. With this perspective in mind, it will be necessary to set up the experiments again to pursue the quantitative comparison.

\section{REFERENCES}

Beardsley, R.C., A laboratory model of the wind driven ocean circulation. J. Fluid Mechanics 1969, 38, $255-271$.

Bleck, R. and Boudra, B., Wind-driven spin-up in eddy-resolving ocean models formulated in isopycnic and isobaric coordinates. J. Geophys. Res 1986, 91, 7611-7621.

Bleck, R., Rooth, C., Hu, D. and Smith, L., Salinity driven thermocline transients in a wind and thermohaline forced isopycnic coordinate model of the North Atlantic. J. Phys. Oceanogr 1992, 22, 1486-1505.

Cessi, P., Laminar separation of colliding western boundary currents. J. Mar. Research 1991, 49, 697-717.

Cessi, P., Ierley, G. and Young, W., A model of the inertial recirculation driven by potential vorticity anomalies. J. Phys. Oceanogr. 1987, 17, 1640-1652.

Cessi, P., Condie, V. and Young, W.R., Dissipative dynamics of western boundary currents. Journal of Marine Research 1990, 48, 677-700.

Charney, J.G., The Gulf Stream as an inertial boundary layer. Proceedings of the National US Academy of Sciences 1955, 41, 731-755.

Chassignet, E.P., Vorticity dissipation by western boundary currents in the presence of outcropping layers. J. Phys. Oceanogr. 1995, 25, 242-255.

Colin de Verdière, A., Quasigeostrophic flows and turbulence in a rotating homogeneous fluid. $\mathrm{PhD}$ thesis, Massachussetts Institute of Technology and Woods Hole Oceanographic Institution 173pp 1977.

Fofonoff, N.P., Steady flow in a frictionless homogeneous ocean. Journal of Marine Research 1954, 13, $254-262$.

Fox-Kemper, B., Reevaluating the role of eddies in multiple wind-driven gyres. J. Phys. Oceanogr. 2005, 35, 1263-1278.

Fox-Kemper, B. and Pedlosky, J., Wind-driven barotropic gyres I: circulation control by eddy vorticity fluxes to an enhanced removal region. J. Mar. Res. 2004, 62, 169-193.

Greenspan, H.P., The theory of rotating fluid, 1969 (Cambridge University Press) 328pp.

Haidvogel, D.B., Mc Williams, J.C. and Gent, P.R., Boundary current separation in a quasigeostrophic, eddy-resolving circulation model. J. Phys. Oceanogr. 1992, 22, 882-902.

Herbette, S., Morel, Y. and Arhan, M., Erosion of a surface vortex by a seamount. J. Phys. Oceanogr. 2003, 33, 1664-1679.

Herbette, S., Morel, Y. and Arhan, M., Surface vortex subduction under an outcropping front. J. Phys. Oceanogr. 2004, 33, 1610-1627.

Herbette, S., Morel, Y. and Arhan, M., Erosion of a surface vortex by a seamount on the $\beta$ plane. J. Phys. Oceanogr. 2005, 35, 2012-2030.

Hide, R., On source sink flows in a rotating fluid. J. Fluid Mech. 1968, 32, 737-764.

Hoskins, B., McIntyre, M. and Robertson, W., On the use and significance of isentropic potential vorticity maps. Quart. J. Roy. Meteor. Soc. 1985, 111, 877-946.

Ierley, G.R. and Young, W.R., Viscous instabilities in the western boundary layer. J. Phys. Oceanogr. 1991, 21, 1323-1332.

Ierley, G., On the onset of inertial recircualtion in barotropic general circulation model. J. Phys. Oceanogr. 1987, 17, 2366-2374.

Kuo, H.L., Dynamics instability of two-dimensional nondivergent flow in a barotropic atmosphere. $J$. Meteor. 1949, 6, 105-122.

Lehoucq, R.B., Sorensen, D.C. and Yang, C., ARPACK User's guide: solution of large 
scale eigenvalue problems with implicitly restarted anorldi methods. SIAM 1998, p. 160pp http://www.caam.rice.edu/software/ARPACK/.

Lozier, S. and Riser, S., Potential vorticity sources and sinks in a quasigeostrophic ocean beyond western boundary currents. J. Phys. Oceanogr. 1990, 20, 1608-1627.

Marshall, J.C., Eddy-mean flow interaction in a barotropic ocean model. Quaterly Journal of the Royal Meteorological Society 1984, 110, 573-590.

Morel, Y. and McWilliams, J., Effects of isopycnal and diapycnal mixing on the stability of oceanic currents. J. Phys. Oceanogr. 2001, 31, 2280-2296.

Moro, B., On the non linear Munk model. I. Steady flows. Dyn. Atmos. Oceans 1988, 12, 259-287.

Moro, B., On the non linear Munk model. II. Stability. Dyn. Atmos. Oceans 1990, 14, 203-227.

Morse, P.M. and Feschbach, H., Methods of theoretical physics, 1st edn, 1953 (Mc Grow-Hill Company).

Munk, W., On the wind-driven ocean circulation. Journal of Meteorology 1950, 7, 79-93.

Pedlosky, J., Geophysical Fluid Dynamics, 2nd edn, 1987 (Springer-Verlag) 710 pp.

Pedlosky, J., Ocean circulation theory, 1st edn, 1996 (Springer) 453pp.

Rhines, P. and Young, W., Homogenization of potential vorticity in planetary gyres. J. Fluid. Mechanics 1982, 122, 347-367.

Rhines, P.B., Geostrophic turbulence. Ann. Review of Fluid Mechanics 1979, 11, 401-441.

Rhines, P.B. and Holland, W., A theoretical discussion on eddy-driven mean flows. Dyn. Atmos. Oceans 1979, 3, 289-325.

Schopp, R. and Colin de Verdière, A., Taylor columns between concentric spheres. Geophys. Astrophys. Fluid Dynamics 1997, 86, 43-73.

Stommel, H., The westward intensification of wind-driven ocean currents; in Transactions of the American Geophysical Union, Vol. 29 1948, pp. 202-206.

Stommel, H., Is the South Pacific helium-3 plume dynamically active?. Earth and Plan. Sci. Lett. 1982, 61, 63-67.

Stommel, H. and Arons, A.B., On the abyssal circulation of the world ocean-I. Stationary planetary flow patterns on a sphere. Deep Sea Research 1961, 6, 140-154.

Stommel, H., Arons, A.B. and Faller, J., Some examples of stationary flow patterns in bounded basins. Tellus 1958, 10, 179-187.

Strogatz, S.H., Nonlinear dynamics and chaos, with applications to physics, biology, chemistry, and engineering, 1994 (Reading, Massachussetts: Perseus books) 498pp.

Verron, J. and Le Provost, C., Response of eddy-resolved general circulation numerical models to asymetrical wind forcing. Dyn. Atm. Ocean 1991, 15, 505-533.

Zalesak, S., Fully multidimensional flux-corrected transport algorithms for fluids. J. Comput. Phys. 1979, 31, 335-362. 